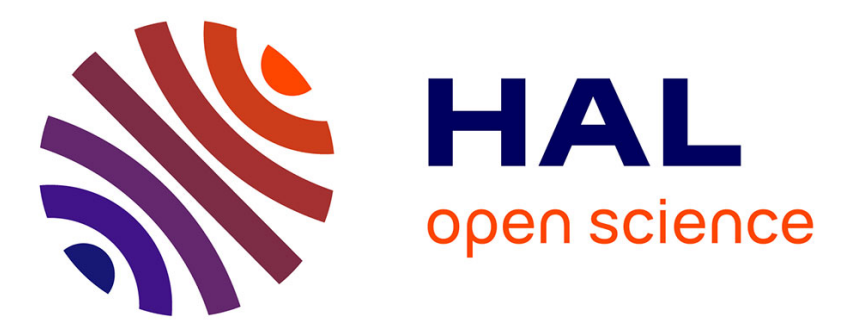

\title{
About samples, giving examples: Optimized Single Molecule Localization Microscopy
} Angélique Jimenez, Karoline Friedl, Christophe Leterrier

\section{To cite this version:}

Angélique Jimenez, Karoline Friedl, Christophe Leterrier. About samples, giving examples: Optimized Single Molecule Localization Microscopy. Methods, 2019, 10.1016/j.ymeth.2019.05.008 . hal02146929

\section{HAL Id: hal-02146929 \\ https://hal-amu.archives-ouvertes.fr/hal-02146929}

Submitted on 4 Jun 2019

HAL is a multi-disciplinary open access archive for the deposit and dissemination of scientific research documents, whether they are published or not. The documents may come from teaching and research institutions in France or abroad, or from public or private research centers.
L'archive ouverte pluridisciplinaire HAL, est destinée au dépôt et à la diffusion de documents scientifiques de niveau recherche, publiés ou non, émanant des établissements d'enseignement et de recherche français ou étrangers, des laboratoires publics ou privés. 


\title{
About samples, giving examples: Optimized Single Molecule Localization Microscopy
}

\author{
Angélique Jimenez ${ }^{\mathrm{a}}$, Karoline Friedl ${ }^{\mathrm{a}, \mathrm{b}}$, Christophe Leterrier ${ }^{\mathrm{a}, *}$ \\ a Aix Marseille Université, CNRS, INP UMR7051, NeuroCyto, Marseille, France \\ ${ }^{\mathrm{b}}$ Abbelight, Paris, France
}

\section{A R T I C L E I N F O}

\section{Keywords:}

Super-resolution microscopy

SMLM

STORM

DNA-PAINT

Cytoskeleton

\begin{abstract}
A B S T R A C T
Super-resolution microscopy has profoundly transformed how we study the architecture of cells, revealing unknown structures and refining our view of cellular assemblies. Among the various techniques, the resolution of Single Molecule Localization Microscopy (SMLM) can reach the size of macromolecular complexes and offer key insights on their nanoscale arrangement in situ. SMLM is thus a demanding technique and taking advantage of its full potential requires specifically optimized procedures. Here we describe how we perform the successive steps of an SMLM workflow, focusing on single-color Stochastic Optical Reconstruction Microscopy (STORM) as well as multicolor DNA Points Accumulation for imaging in Nanoscale Topography (DNA-PAINT) of fixed samples. We provide detailed procedures for careful sample fixation and immunostaining of typical cellular structures: cytoskeleton, clathrin-coated pits, and organelles. We then offer guidelines for optimal imaging and processing of SMLM data in order to optimize reconstruction quality and avoid the generation of artifacts. We hope that the tips and tricks we discovered over the years and detail here will be useful for researchers looking to make the best possible SMLM images, a pre-requisite for meaningful biological discovery.
\end{abstract}

\section{Introduction}

Optical microscopy of immunofluorescence-labeled samples has revolutionized biology by allowing access to cellular processes in their native setting. However, the resolution of an optical microscope is physically limited to about $200 \mathrm{~nm}$ due to the diffraction of light that occurs along the optical path $[1,2]$. This limit prevents the detailed visualization of key cellular structures: organelles (mitochondria, endosomes), cytoskeleton assemblies (actin, microtubules, intermediate filaments) and other scaffolding structures such as clathrin-coated pits that all have typical dimensions between 10 and $500 \mathrm{~nm}$ [3]. New optical techniques, collectively called super-resolution microscopy, can now overcome this limit and resolve details down to a few tens of nanometers [4]. Since its emergence in the beginning of the 2000s, super-resolution microscopy has matured and is now used in many laboratories to investigate the nanoscale cellular architecture $[5,6]$.

Among the widely available super-resolutive techniques, Single Molecule Localization Microscopy (SMLM) is the one that can attain the finest precision, getting close to ultrastructural details of a few nanometers in the best cases [7]. SMLM obtains information beyond the diffraction limit by pinpointing the position of single fluorophores, a mechanism that is distinct from other strategies such as STimulated
Emission Depletion (STED) or Structured Illumination Microscopy (SIM) [2]. Experimental comparisons of super-resolution methods can be found in $[8,9]$. Due to diffraction by the microscope objective, a single fluorophore emitting light will appear as a $\sim 200 \mathrm{~nm}$-wide spot called the point-spread function (PSF). However, if the fluorophore is well isolated, it is possible to fit its position with a precision well beyond the size of the PSF (Fig. 1A). This localization precision primarily depends on the number of photons emitted by the fluorophores and is typically around $\sim 8 \mathrm{~nm}$ for standard deviation (s.d., $20 \mathrm{~nm}$ in FWHM) $[10,11]$. Single-molecule localization was thus used since the 1980 s to track the movement of single particles and applied to fluorescence imaging to follow the diffusion of membrane proteins [12] or the movement of molecular motors [13].

To take advantage of single-molecule localization and generate an image of a fluorescently labeled sample, it is necessary to separate the PSF from each fluorophore, as initially proposed by Betzig [14]. SMLM separates fluorophores along the temporal dimension by photoswitching: each fluorophore labeling the sample is active only very briefly, emitting fluorescence as one or several short spontaneous blinking events. At any given time, most fluorophores are dark and only a few of them are blinking, allowing to localize them by fitting their position (Fig. 1B). The blinking of fluorophores is generally acquired as

\footnotetext{
* Corresponding author at: Faculté de Médecine - Secteur Nord, Aix-Marseille Université, Bd Pierre Dramard, 13344 Marseille Cedex 15, France.

E-mail address: christophe.leterrier@univ-amu.fr (C. Leterrier).
} 


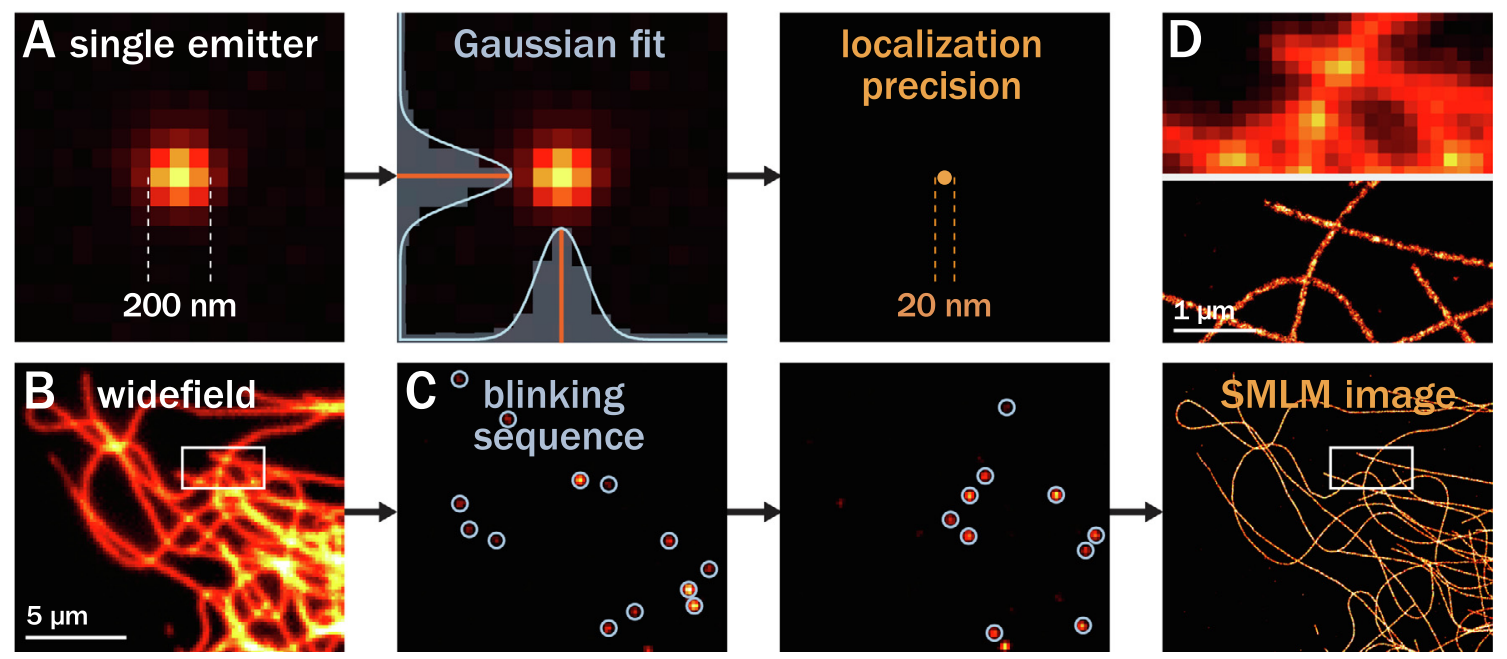

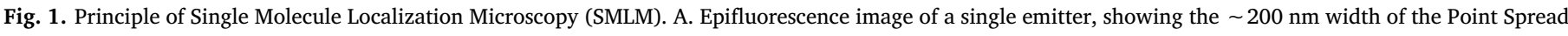

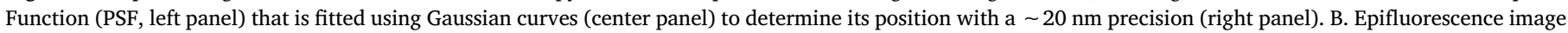

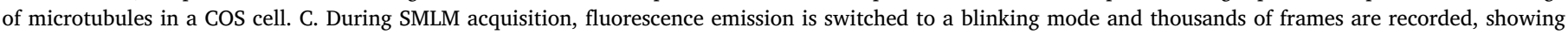

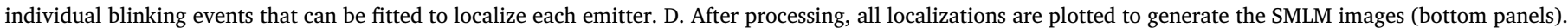
Top panel is a zoom corresponding to the box highlighted in the full image and shows the gain in resolution with much thinner microtubules (top panels).

a long sequence of thousands of frames, in order to localize millions of fluorophores. The SMLM image is then reconstructed by plotting all the localized fluorophores, resulting in a super-resolved image that has a $\sim 10 \mathrm{X}$ better resolution than the diffraction-limited image (Fig. 1C).

The mechanism used to generate blinking fluorophores distinguishes several techniques that belong to SMLM: PALM, STORM and DNA-PAINT. In Photoactivated Localization Microscopy (PALM), photoactivatable or photoconvertible fluorescent proteins are used and blinking is generated by illuminating the sample to sparsely activate or convert these fluorescent proteins $[15,16]$. One advantage of PALM is that it can be performed on living cells expressing photoactivatable protein fusions. This notably allows to access the dynamics of single proteins, in a variant called sptPALM [17]. In STochastic Optical Reconstruction Microscopy (STORM), high intensity illumination and an oxygen-deprived, reducing buffer is generally used to induce sparse blinking of organic fluorophores commonly used for immunolabeling $[18,19]$. Single-color STORM is readily compatible with classical immunolabeled samples, by using Alexa Fluor 647-coupled secondary antibodies [20]. Multi-color STORM is not as straightforward, as it is a challenge to find two distinct fluorophores that have good blinking characteristics in the same environment, and inducing photoswitching of fluorophores outside of the far-red channel usually require high laser power illumination [21,22]. In DNA Points Accumulation for imaging in Nanoscale Topography (DNA-PAINT) [23,24], blinking is generated by the transient hybridization of short DNA single-strand coupled to a fluorophore (imager strand) with its complementary strand (docking strand) attached to an antibody targeting the structure of interest $[25,26]$. DNA-PAINT does not require a specific buffer or very high laser power, as the blinking does not depend on the photophysics of the fluorophore. Moreover, fluorophores are constantly renewed at the sample as new imagers interact with the docking strands, allowing to accumulate a large number of localizations. Finally, multi-color DNAPAINT is straightforward by using orthogonal docking strands on distinct secondary antibodies and corresponding imagers, allowing to image 5-8 different targets [27-30].

Since their invention, these different SMLM techniques have been extensively used to probe the nanoscale arrangement of cellular structures and macromolecular complexes [31,32]. Nevertheless, SMLM is a challenging technique that requires specific knowledge and skills in order to obtain good quality data [33]. We have been using STORM since 2013, then DNA-PAINT to study the organization of the axonal cytoskeleton [34,35], resolving the architecture of axonal actin and spectrins [36-38] as well as the mechanisms of slow axonal transport $[39,40]$. We have also helped developing SMLM imaging modalities [29] and analysis strategies [41,42]. During these years, we have refined our SMLM workflow by optimizing sample preparation, imaging and analysis. In this Methods article, we aim at summarizing our experience to help researchers that use SMLM or are interested in trying it. We will provide advice and tips along the whole workflow of cell culture, fixation, immunolabeling, imaging and processing (Fig. 2). We will describe how to prepare good reference test samples for STORM and DNA-PAINT by labeling abundant targets in fibroblasts cells (Fig. 2A and B). Cytoskeleton (actin and microtubules) is a good SMLM benchmark target, as it forms well-known cellular patterns and can be labeled at high density. Other targets include clathrin-coated pits, which $\sim 100 \mathrm{~nm}$ size is ideal to verify and benchmark the proper performance of an SMLM setup. We will summarize the specific procedures and tweaks we use during imaging and briefly explain our processing workflow (Fig. 2C and D). This will complement the existing reviews and methodological articles on how to perform STORM and DNAPAINT [20,43-48]. We hope this will be useful to the microscopy community and help interested researchers to get the best possible quality for their SMLM images.

\section{Sample preparation}

\subsection{Cell culture}

Flat and large cells make nice sample for SMLM, as they provide areas of thin cytoplasm rich in cytoskeletal and scaffolding elements. We are using COS-7 fibroblasts from Green African Monkey (ATCC, ref. CRL-1651) that are easy to culture and grow rapidly [49], but other mammalian cell lines are also typically used such as U2OS [50], BS-C-1 $[51,52]$, or HeLa, which are thicker and can be more challenging to image [53]. It is important to know a bit about the underlying biology of the chosen cell line (use as a biological model, tumor origin...) in order to understand the possible singularities in their organization. For example, COS-7 cells frequently fail to properly divide and have a significant population of multi-nucleated cells. Sterile technique and health and safety regulations require a dedicated space for cell culture, and particular care must be taken to avoid contamination, notably by mycoplasmas, and by other cell lines leading to cell mis-identification 


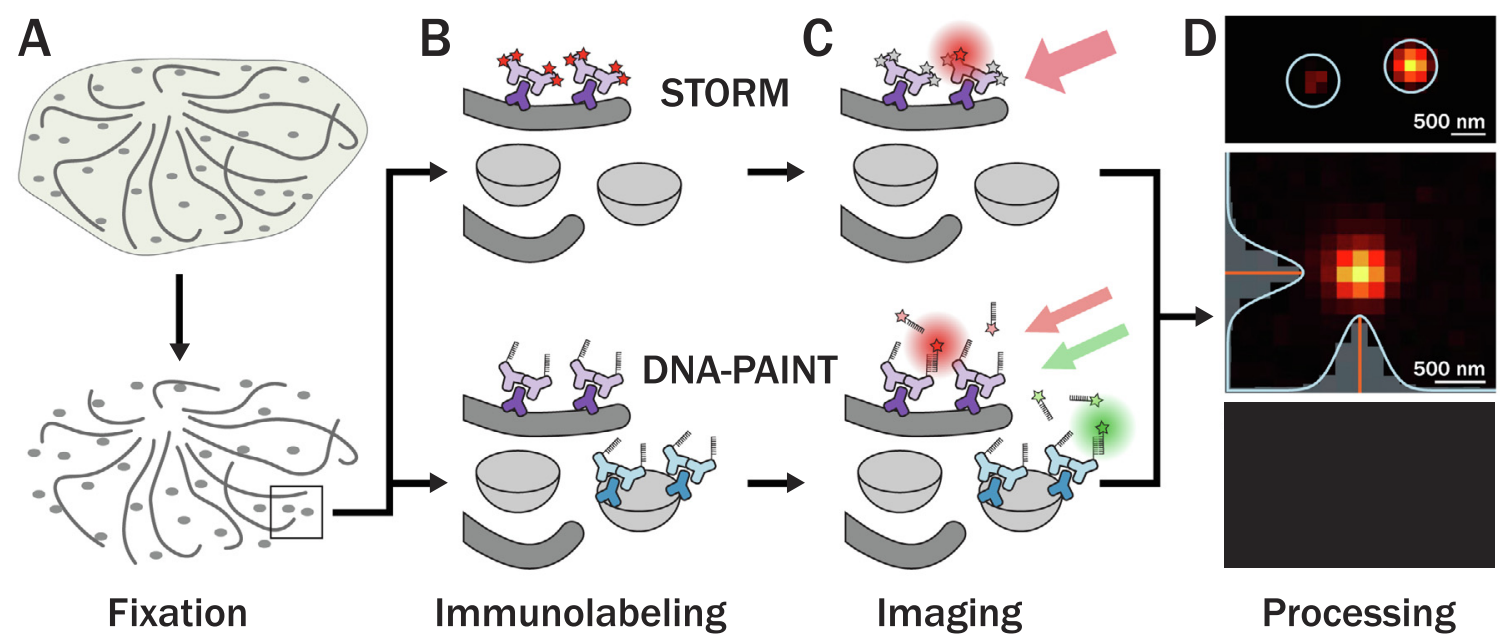

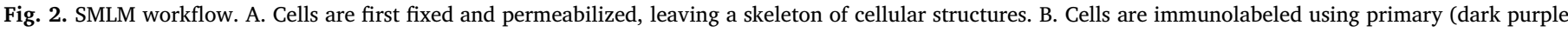

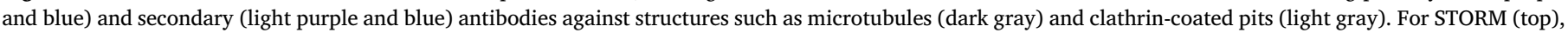

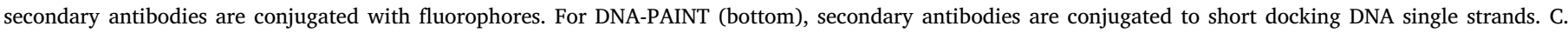

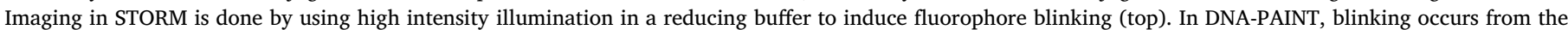

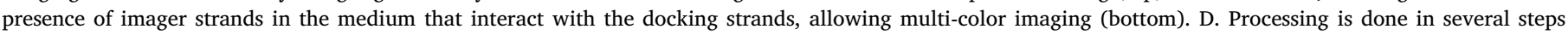

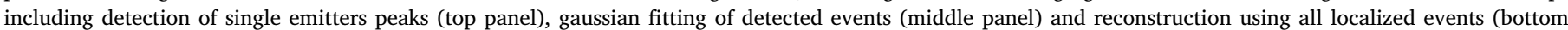

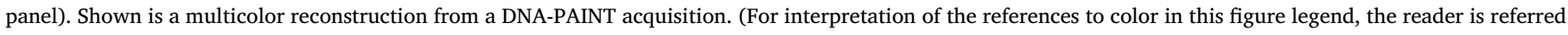
to the web version of this article.)

[54]. Proper cell culture technique should allow cells to grow over tens of passages without the need for constant antibiotic presence in the culture medium, and the culture should be regularly re-started from frozen stocks to avoid genetic drift and change in their properties.

\subsection{Cell seeding}

The first step in SMLM sample preparation is to seed cells on glass coverslips. The best optical quality is obtained by using coverslips of $0.17 \mathrm{~mm}$ thickness, known as \#1.5, for which high numerical aperture oil objectives (60X to 100X) typically used in SMLM are optimized. Some aspects of SMLM, notably 3D calibration, are highly dependent on coverslip thickness. In order to minimize variation from the calibrated curve, it is good to use high-precision thickness coverslips which have a thickness tolerance of $175 \pm 5 \mu \mathrm{m}$ rather than $175 \pm 15 \mu \mathrm{m}$. We use 18-mm diameter round coverslips (Dutscher, ref. 900556) that are well adapted to our chambers and sample mounting options (see below). Coverslips should be thoroughly cleaned by successive baths: we clean our coverslips in racks using successive nitric acid, water and absolute ethanol baths followed by $2 \mathrm{~h}$ at $180^{\circ} \mathrm{C}$ [55]. Once cleaned, coverslips are treated with poly-L-lysine to favor cell attachment (Sigma, ref. P2636); clean coverslips should become hydrophilic, with an easy spreading of the polylysine solution over the whole coverslip. After rinses, cells are seeded to $10-20 \%$ confluency and incubated overnight for attachment and spreading. A low confluency helps obtaining a significant proportion of single cells after $24 \mathrm{~h}$ that will give the best results by SMLM.

\subsection{Calibration coverslips}

When preparing coverslips for cell seeding, we usually prepare a couple of extra coverslips, up to the polylysine treatment, that we subsequently store in phosphate buffer. These are used to prepare sister calibration coverslips by just incubating them 5 min with $0.1 \mu \mathrm{m}$ Tetraspeck beads (Thermo Fisher, ref. T7279) diluted 1:100-1:800 depending on the desired density of beads. Beads coverslips are notably useful for 3D-STORM calibration (see below), to verify the proper alignment of the laser and field homogeneity or calibrate chromatic aberration correction as they are fluorescent in the commonly used channels (488, 561 and $647 \mathrm{~nm}$ lasers).

\section{Immunostaining}

\subsection{Fixation}

Immunocytochemistry usually begins with the fixation of cells using chemical fixatives. An optimized fixation protocol is crucial to minimize artifacts that will be revealed at the nanoscale [56]. For example, cold methanol fixation is widely used before staining for microtubules [57], but in our experience results in poor preservation of microtubule organization at the nanoscale. As a rule of thumb, methods that have been validated at the ultrastructural level in electron microcopy sample preparation protocols are most likely to give the best results. As described previously [56], we found that washing steps should be avoided before fixation. For the cytoskeleton, the best fixation uses glutaraldehyde in a cytoskeleton-preserving buffer. This can be preceded by a quick extraction step that will remove soluble proteins just before fixation, notably actin and tubulin monomers. Our pre-extraction/ fixation protocol uses a moderate concentration of glutaraldehyde. It is optimal for microtubules and actin labeling but is also compatible with imaging of clathrin-coated pits (Figs. 3A, 4, 5, 6A and 7). After glutaraldehyde-based fixation, a quenching step using $\mathrm{NaBH}_{4}$ (Sigma, ref. 213462) as a reducing agent is necessary and works better than the $\mathrm{NH}_{4} \mathrm{Cl} /$ glycine incubation that is sometimes used.

\section{Glutaraldehyde fixation protocol}

PEM = 80 mm PIPES, 5 mm EGTA, 2 mm $\mathrm{MgCl}_{2}$, pH 6.8 .

Extraction solution: $0.25 \%$ Triton, $0.1 \%$ glutaraldehyde in PEM, pre-heated to $37^{\circ} \mathrm{C}$.

Fixation solution: $0.25 \%$ Triton, $0.5 \%$ glutaraldehyde in PEM, preheated to $37^{\circ} \mathrm{C}$.

Work at $37^{\circ} \mathrm{C}$ using a water bath or pre-heated pad for the extraction and fixation steps.

- Incubate with extraction solution for 15-45 s.

- Replace with fixation solution and incubate for $10 \mathrm{Min}$.

- Replace with $0.1 \% \mathrm{NaBH}_{4}$ in phosphate buffer and incubate 7 Min at room temperature. 

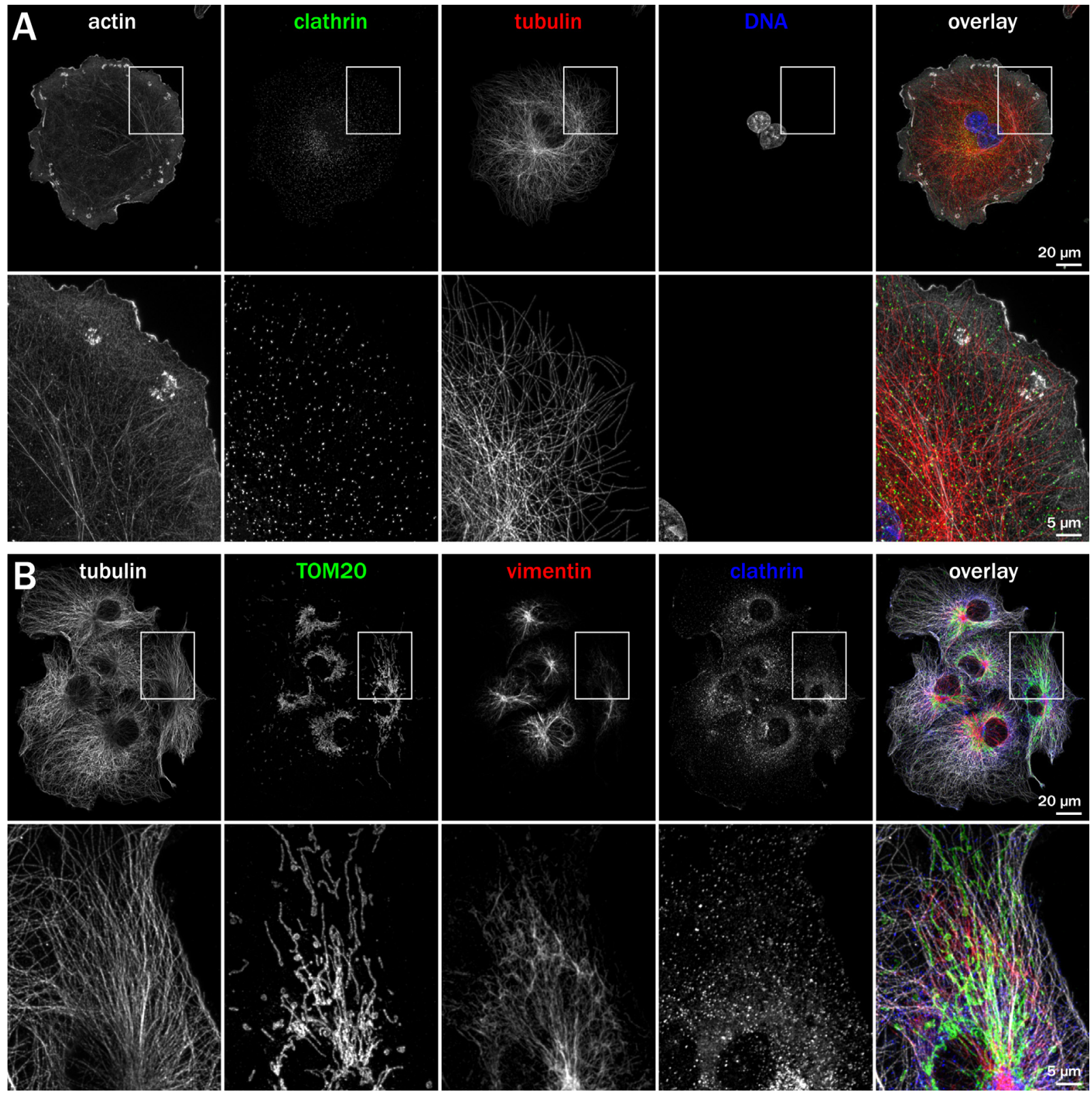

Fig. 3. Diffraction-limited images of immunostained COS cells. Images are projections of deconvolved Z-stacks, acquired with an Apotome microscope (Zeiss) equipped with a 63X, NA 1.4 objective and a Flash4 v3 sCMOS camera (Hamamatsu). A. COS cell fixed using the glutaraldehyde pre-extraction/fixation protocol, stained for actin (phalloidin, gray on overlay), microtubules (two anti- $\alpha$-tubulin antibodies, green on overlay), clathrin (anti-clathrin heavy chain, red on overlay) and DNA (DAPI, blue on overlay). Bottom panels are zooms corresponding to the box highlighted on the full image (top panels). B. COS cells fixed using the hot paraformaldehyde fixation protocol, stained for microtubules (anti-tubulin, gray on overlay), intermediate filaments (anti-vimentin, red on overlay), mitochondria (anti-TOM20, green on overlay) and clathrin (anti-clathrin heavy chain, blue on overlay). Bottom panels are zooms corresponding to the box highlighted on the full image (top panels). (For interpretation of the references to color in this figure legend, the reader is referred to the web version of this article.)

- Rinse two times quickly with phosphate buffer.

The glutaraldehyde-based fixation protocol will not allow optimal labeling for other targets such as mitochondria (TOM20/TOM22 protein) or intermediate filaments (vimentin). Alternatively, we use formaldehyde (FA) in cytoskeleton preserving buffer that works also very well for actin and clathrin [58]. If used at $37^{\circ} \mathrm{C}$, this protocol can lead to satisfactory microtubule preservation (Figs. 3B, 7B). However, in our hands it is not robust and not as good as the glutaraldehyde-based fixation. Quenching after FA-based fixation is usually not necessary and does not provide a better immunolabeling. An important point for optimal sample preparation is the freshness of the aldehydes used for fixation: we use electron-microscopy grade glutaraldehyde (25\%,
Sigma, ref. G5882) and formaldehyde (37\%, EMS Diasum, ref. 15714) in pure water, in glass ampoules. After opening, they are stored at $4{ }^{\circ} \mathrm{C}$ and used within two weeks.

\section{FA-PEM fixation protocol}

PEM $=80$ mM PIPES, 5 mM EGTA, $2 \mathrm{mM} \mathrm{MgCl}_{2}$, pH 6.8 .

Fixation solution: 4\% FA, 4\% sucrose in PEM.

- Fix for $10 \mathrm{~min}$ at room temperature or use fixation solution preheated to $37^{\circ} \mathrm{C}$.

- Rinse three times quickly with phosphate buffer. 


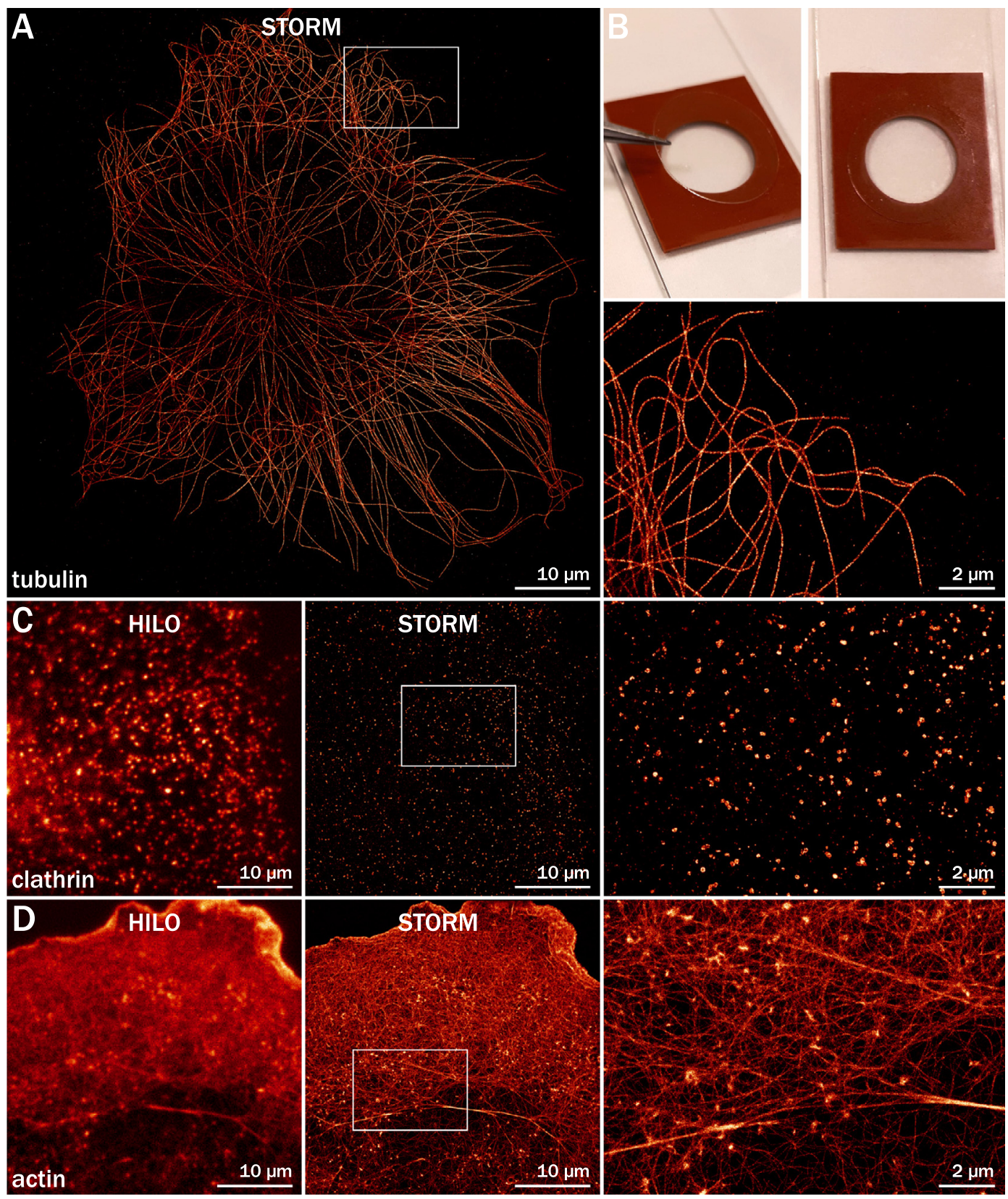

Fig. 4. 2D STORM images of microtubules, clathrin and actin in COS cells. A. STORM image of a COS cell labeled for microtubules (two anti- $\alpha$-tubulin antibodies), acquired with an sCMOS camera $(80 \times 80 \mu \mathrm{m}$ field of view). Right panel is a zoom of the area highlighted on the full image. B. Photograph showing the glass slideattached silicone chamber (red) on which the $18 \mathrm{~mm}$ coverslip is sealed by gentle pressure (left) to obtain a closed chamber filled with STORM buffer (right). C. Diffraction-limited HILO image (left panel) and corresponding STORM image (center panel) of a COS cell labeled for clathrin-coated pits (anti-clathrin light chain), acquired with an EMCCD camera ( $40 \times 40 \mu \mathrm{m}$ field of view). Right panel is a zoom of the area highlighted on the full image. D. Diffraction-limited HILO image (left panel) and corresponding STORM image (center panel) of a COS cell labeled for actin (phalloidin-AF647). Right panel is a zoom of the area highlighted on the full image. (For interpretation of the references to color in this figure legend, the reader is referred to the web version of this article.)

\subsection{Blocking and primary antibodies incubation}

We found blocking to be an important step, and usually perform it for at least $1 \mathrm{~h}$ at room temperature (and up to $3 \mathrm{~h}$ when staining clathrin) with gentle agitation. We use gelatin as the blocking agent (Sigma, ref. G9391), and permeabilize the cells at the same time using $0.1 \%$ Triton X-100 (Sigma, ref. T8787). This blocking buffer (phosphate

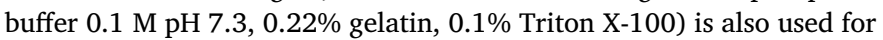
primary and secondary antibodies incubation. After blocking and permeabilization, we perform antibody incubations and rinses inside blackened-out large square Petri dishes, on Parafilm (Table 1). This allows to use a minimal amount of reagents: for antibodies and phalloidin incubations, $18 \mathrm{~mm}$ coverslips are flipped (cells facing downward) on $50 \mu \mathrm{L}$ droplets and are flipped back (cells facing upward) for rinses.

For SMLM, it is important to maximize antibody coverage, i.e. ensure that the target epitopes are labeled with the highest possible efficiency. This entails using higher concentration of primary antibodies as long as this does not lead to overwhelming background (for example, anti-tubulin antibodies can be used at 10X the concentration used for regular epifluorescence labeling, Table 1). Moreover, higher labeling density for a given structure can be obtained by using several antibodies against different epitopes within this structure. This strategy has been used to maximize signal for synapses [59] and microtubules in SMLM 

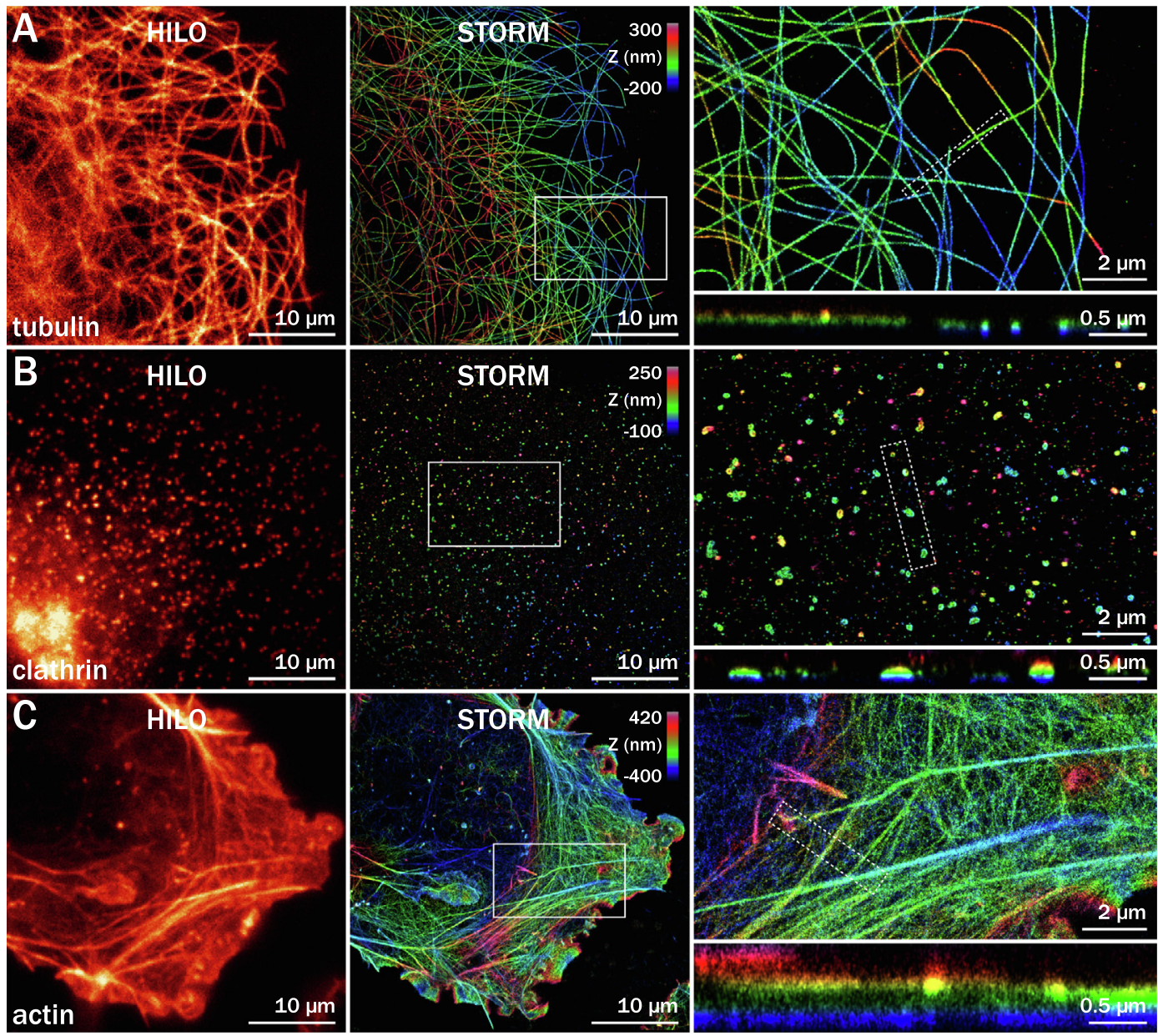

Fig. 5. 3D STORM images of microtubules, clathrin and actin in COS cells. A. Diffraction-limited HILO image (left panel) and corresponding astigmatism-based 3D STORM image (center panel) of a COS cell labeled for microtubules (two anti- $\alpha$-tubulin antibodies), color-coded for depth. Top right panel is a zoom of the area highlighted on the full image, bottom right panel shows an XZ cross-section along the line highlighted on the zoomed image. B. Diffraction-limited HILO image (left panel) and corresponding 3D STORM image (center panel) of a COS cell labeled for clathrin-coated pits (anti-clathrin light chain), color-coded for depth. Top right panel is a zoom of the area highlighted on the full image, bottom right panel shows an XZ cross-section along the line highlighted on the zoomed image. C. Diffraction-limited HILO image (left panel) and corresponding 3D STORM image (center panel) of a COS cell labeled for actin (phalloidin-AF647), color-coded for depth. Top right panel is a zoom of the area highlighted on the full image, bottom right panel shows an XZ cross-section along the line highlighted on the zoomed image.

[50] or Expansion Microscopy [53]. Similarly, we usually label microtubules with a combination of anti- $\alpha$ - and anti- $\beta$-tubulin monoclonal antibodies to obtain a dense labeling in STORM and PAINT (Fig. 3). Finally, in our hands the highest labeling density is obtained using overnight incubation of the primary antibody mixture at $4{ }^{\circ} \mathrm{C}$. In any case, it is always useful to optimize primary antibody incubation parameters (concentration, temperature, duration) depending on the type of sample and target.

\subsection{Secondary antibodies for STORM and PAINT}

After primary antibodies incubation, the coverslips are rinsed using blocking buffer (3x10 minutes), then the cells are incubated with secondary antibodies in blocking buffer for $1 \mathrm{~h}$ at room temperature. For STORM, we use commercial full IgG secondary antibodies coupled to Alexa Fluor 647 (Table 2) at a concentration of 4-7 $\mu \mathrm{g} / \mathrm{mL}$ in blocking buffer. It is possible to co-stain cells using other antibodies revealed by different fluorophores such as Alexa Fluor 488 and Alexa Fluor 555. This allows to locate cells and interesting structures or complement the STORM image with diffraction-limited epifluorescence images from other targets. It is particularly useful for DNA-PAINT, because the DNAcoupled secondary antibodies do not provide steady-state fluorescence allowing to locate cells and features of interest. In this case, it is possible to label the same structure with an antibody against a distinct epitope and a fluorescent secondary antibody.

Labeling with primary and secondary antibodies (each with a $\sim 15$ $\mathrm{nm}$ size) adds a linkage error corresponding to the distance between the epitope and the fluorophore. In the case of microtubules, as antibodies can only go outward, this leads to a larger apparent diameter of $\sim 60$ $\mathrm{nm}$ (versus $25 \mathrm{~nm}$ for the real external microtubule diameter). A solution to minimize linkage error is to use directly labeled primary antibodies or nanobodies [60]. However, the use of direct labeling or nanobodies will result in less signal amplification and thus a reduced number of fluorophores and localizations [61], which can make the final structure appear spottier. In the general case where antibodies can bind on any side of the epitope, primary and secondary antibodies each add an uncertainty of s.d $\sim 6.5 \mathrm{~nm}$ around the epitope position, which is then combined with the localization precision of the fluorophore. For typical STORM localization precision (s.d. $\sim 7 \mathrm{~nm}$ ), this leads to a moderate degradation of the localization precision to s.d. $\sim 11 \mathrm{~nm}$ (FWHM from 16 to $25 \mathrm{~nm}$ ) [38,62].

For multicolor imaging using the Exchange-PAINT variant of DNAPAINT [25], we use specific secondary antibodies coupled to 9-basepair single strand of DNA (the "docking" strand). These DNA-coupled 

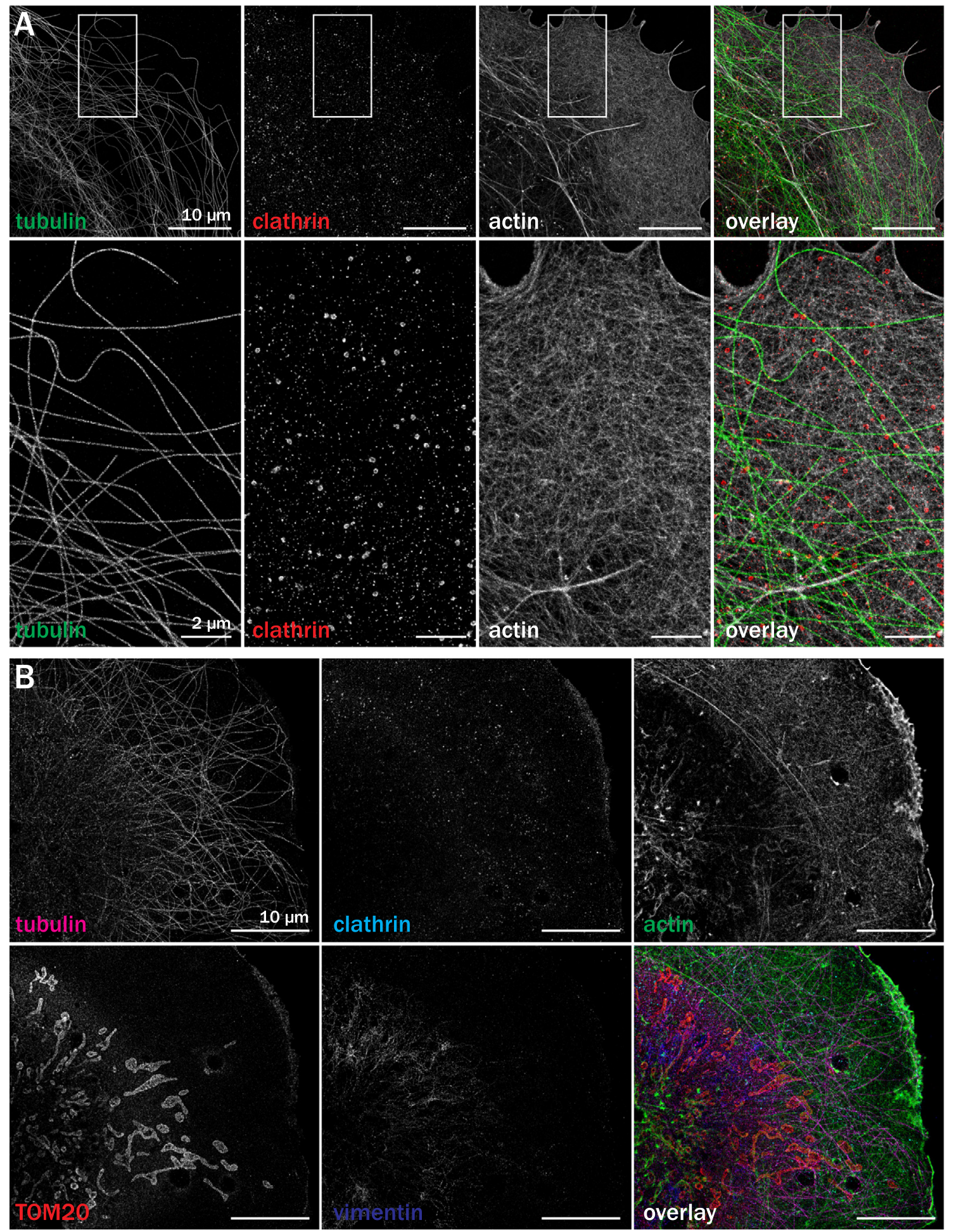

Fig. 6. Multicolor STORM and DNA-PAINT images of immunostained COS cells. A. 3-color image obtained by sequential acquisition of actin by STORM (phalloidinAF647, gray in overlay), microtubules (two anti- $\alpha$-tubulin antibodies, green on overlay) and clathrin-coated pits (anti-clathrin light chain, red on overlay) by DNAPAINT (Atto655 imager). Bottom panels are zoomed images corresponding to the highlighted area on the full image. B. 5-color image obtained in three steps: first DNA-PAINT of actin (phalloidin-Atto488, green in overlay), microtubules (two anti- $\alpha$-tubulin antibodies, Atto565 imager, magenta on overlay) and mitochondria (anti TOM20, Atto655 imager, red on overlay), then DNA-PAINT of intermediate filaments (anti-vimentin, Cy3B imager, blue on overlay), then DNA-PAINT of clathrin (anti-clathrin light chain, Су3B imager, cyan on overlay). (For interpretation of the references to color in this figure legend, the reader is referred to the web version of this article.)

antibodies are not available commercially but can be prepared within two days in a biology lab thanks to published coupling protocols and sequences [48]. We are using the thiol coupling protocol as described in [48] to conjugate donkey anti-mouse and donkey anti-rabbit antibodies with the P1 (ATACATCTA) and P3 (TCTTCATTA) docking strands, respectively. These secondary antibodies are tested for optimal concentration, which is usually 1:50-1:100 and incubated for $1 \mathrm{~h}$ at room temperature in blocking buffer, similar to fluorescent secondary 

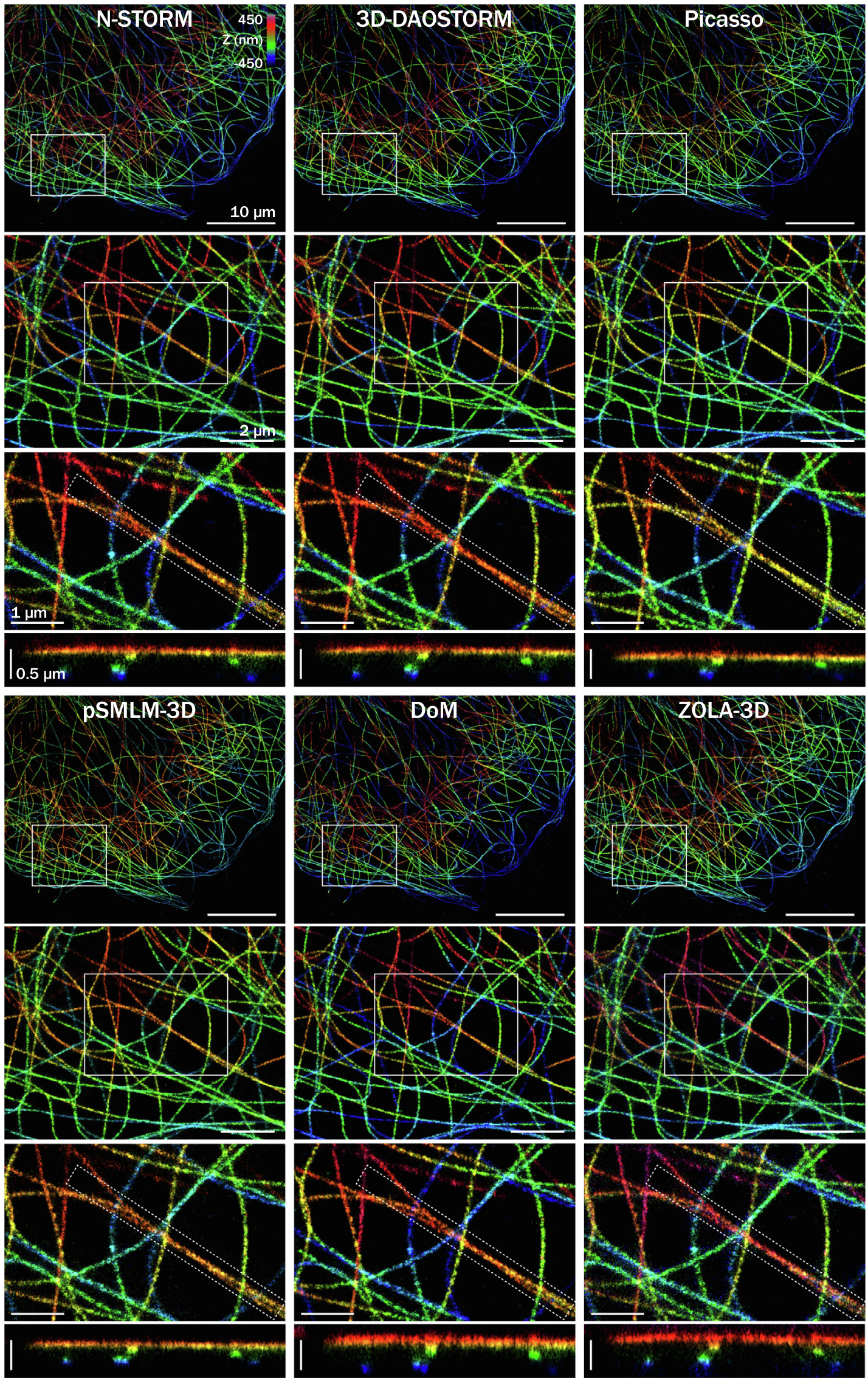

Fig. 7. Processing of 3D-STORM data using several examples of SMLM software. COS cell labeled for microtubules (two anti- $\alpha$-tubulin antibodies) and imaged by 3D STORM. The acquired image sequence has been processed using different software packages using optimized parameters for each software: N-STORM (Nikon, proprietary); 3D-DAOSTORM (open source, Python); Picasso (open source, Python); phasor pSMLM-3D (open source, ImageJ); DoM Utrecht (open source, ImageJ); ZOLA-3D (open source, ImageJ). See Table 3 for more information about each software. 
Table 1

Primary antibodies used.

\begin{tabular}{|c|c|c|c|c|c|c|c|}
\hline Structure & Target protein & Species & Ig type & Clone & Supplier & Cat \# & Dilution \\
\hline microtubules & $\alpha$-tubulin & rabbit polyclonal & - & - & abcam & ab18251 & $1: 300$ \\
\hline microtubules & $\alpha$-tubulin & mouse monoclonal & IgG1 & B-5-1-2 & Sigma & T5168 & $1: 300$ \\
\hline microtubules & $\alpha$-tubulin & mouse monoclonal & IgG1 & DM1A & Sigma & T6199 & $1: 300$ \\
\hline microtubules & ß-tubulin & mouse monoclonal & IgG1 & $2-28-33$ & Sigma & T5293 & $1: 200$ \\
\hline clathrin-coated pits & clathrin heavy chain & rabbit polyclonal & - & - & abcam & ab21679 & $1: 150$ \\
\hline microtubules & $\alpha$-tubulin & rat monoclonal & - & YOL1/34 & abcam & ab6161 & $1: 150$ \\
\hline microtubules & tyrosinated $\alpha$-tubulin & rat monoclonal & - & YL1/2 & abcam & ab6160 & $1: 150$ \\
\hline intermediate filaments & vimentin & chicken polyclonal & $\operatorname{IgY}$ & Poly 29,191 & BioLegend & 919,101 & $1: 1000$ \\
\hline mitochondria & TOM20 & mouse monoclonal & IgG1 & 29/TOM20 & BD Bioscience & 612,278 & $1: 200$ \\
\hline
\end{tabular}

antibodies. Extra care should be taken to store the DNA-coupled antibodies, as the linked DNA strand tend to hydrolyze with time. We flashfreeze them in small aliquots just after coupling and keep the currently used aliquot at $4{ }^{\circ} \mathrm{C}$, using it within $\sim 1$ Month. After incubation, secondary antibodies are rinsed once with blocking buffer and twice with phosphate buffer (10 Min each time).

\subsection{Actin staining}

The best results for SMLM of actin are obtained using fluorescent phalloidin, because it allows for a very high density of labeling and results in crisp reconstruction of filamentous actin [63]. As an alternative, PAINT of actin has been performed using phalloidin coupled to a DNA docking strand [28] or actin-targeting affimers [64]. It is also possible to use fluorescent LifeAct that transiently binds to actin in fixed samples to generate an SMLM image, a technique called Integrating exchangeable single-molecule localization (IRIS) [65-67]. In our hands, these alternatives are significantly slower than STORM with fluorescent phalloidin to generate a high-quality image.

For STORM, the best results are obtained using phalloidin-Alexa Fluor 647 (AF647, Thermo Fisher or Cell Signaling Technologies). An important point is that phalloidin-AF647 is more labile than uncoupled phalloidin or phalloidin coupled to other fluorophores, and tend to detach easily from actin, as noted previously [63]. As a consequence, it is necessary to perform actin labeling as the very last step in the staining protocol. After secondary antibody rinses, cells are incubated in highly-concentrated phalloidin-AF647 $(500 \mathrm{~nm})$ in phosphate buffer, either for $>1 \mathrm{~h}$ at room temperature or overnight at $4{ }^{\circ} \mathrm{C}$. They are kept in phalloidin-AF647 until being imaged. When performing multi-color imaging with DNA-PAINT targets together with actin, we usually label actin with phalloidin-AF647 that gives the best results. Alternatively, we use phalloidin-Atto488 (500 nm phalloidin-Atto488 in phosphate buffer for $>1 \mathrm{~h}$ at room temperature) that can blink in PAINT imaging buffer (see below).

When followed precisely, this fixation and immunostaining protocol provides crisp and bright labeling of cellular structures. To verify the quality of sample preparation, we usually label sister coverslips with fluorescent secondary antibodies. After mounting on glass slides (Prolong Glass, Thermo Fisher), they can be imaged using regular epifluorescence or confocal microscopy to document the experiment (Fig. 3).

\section{Imaging}

\subsection{Hardware setup}

We perform SMLM using a Nikon N-STORM microscope equipped with a 100X, NA 1.49 TIRF objective, a Perfect Focus System for Z focus stabilization, an Andor 512x512 EMCCD camera and an Agilent laser launch (405 nm/25 mW, $488 \mathrm{~nm} / 85 \mathrm{~mW}, 561 \mathrm{~nm} / 85 \mathrm{~mW}, 647 \mathrm{~nm} /$ $125 \mathrm{~mW}$ output). A $2 \mathrm{X}$ lens in the TIRF coupling arm focuses the laser to illuminate the center of the field of view, and SMLM acquisition is performed using the center $256 \times 256$ pixels $(41 \times 41 \mu \mathrm{m}, 160 \mathrm{~nm}$ pixel size). A pixel size of $100-160 \mathrm{~nm}$ is generally used, corresponding to $\leq 1$ s.d. of the PSF for reliable localization of single emitters [10]. The filter cube for STORM has four excitation bands corresponding to the 405, 488, 561 and $647 \mathrm{~nm}$ laser, and a single far-red emission band $(670-760 \mathrm{~nm})$. When combining DNA-PAINT with STORM of actin labeled with phalloidin-Atto 488, we use a 4-band cube that has excitation and emission bands for each laser wavelength. Alternatively, we use a second setup consisting in an Abbelight module on an Olympus stand equipped with a 100X, NA 1.49 objective, Hamamatsu $2048 \times 2048$ sCMOS camera and an Oxxius laser launch (405 nm/100 MW, $488 \mathrm{~nm} / 500 \mathrm{~mW}, 640 \mathrm{~nm} / 500 \mathrm{~mW}$ ). The larger sensor size allows for a smaller pixel size $(97 \mathrm{~nm})$ and a larger field of view $(80 \times 80 \mu \mathrm{m}$, Fig. 4A), requiring higher laser power to induce the fluorophore blinking necessary for STORM.

For optimal SNR, we use the laser illumination in the critical angle fluorescence microscopy mode [68,69], also called highly inclined and laminated optical sheet (HILO) [70] or grazing incidence [71]. This consists in the inclination of the laser angle until reaching the critical angle, a point where the sample is illuminated by a $\sim 1-2 \mu \mathrm{m}$ thick horizontal light sheet above the coverslip, avoiding fluorescence from the upper part of the cell and imaging medium. This is perfectly adapted to the flat parts of the COS cells cytoplasm, and to the $\mathrm{Z}$ range of the astigmatism-based 3D SMLM $(\sim 800 \mathrm{~nm})$. In addition, intensity shows a $\sim 2 \mathrm{X}$ peak precisely at the critical angle [72] which further enhances signal and allows to precisely locate the critical angle using

Table 2

Secondary antibodies used.

\begin{tabular}{|c|c|c|c|c|c|c|c|}
\hline Application & Host species & Target species & Fluorophore & Supplier & Cat \# & Dilution & Concentration \\
\hline STORM & donkey & mouse & Alexa Fluor 647 & Thermo Fisher & A31571 & $1: 300$ & $6.5 \mu \mathrm{g} / \mathrm{mL}$ \\
\hline STORM & donkey & rabbit & Alexa Fluor 647 & Jackson ImmunoResearch & $711-605-152$ & $1: 300$ & $5 \mu \mathrm{g} / \mathrm{mL}$ \\
\hline DNA-PAINT & donkey & mouse & PAINT P1 sequence & Homemade & & $1: 100$ & \\
\hline DNA-PAINT & donkey & rabbit & PAINT P3 sequence & Homemade & & $1: 100$ & \\
\hline epifluorescence & donkey & rabbit & Dylight 405 & Rockland & $611-746-127$ & $1: 300$ & $1.3 \mu \mathrm{g} / \mathrm{mL}$ \\
\hline epifluorescence & donkey & mouse & Alexa Fluor 488 & Invitrogen & A21202 & $1: 300$ & $6.7 \mu \mathrm{g} / \mathrm{mL}$ \\
\hline epifluorescence & goat & rat & Alexa Fluor 555 & Invitrogen & A21434 & $1: 400$ & $5 \mu \mathrm{g} / \mathrm{mL}$ \\
\hline epifluorescence & donkey & rabbit & CF568 & Biotium & 20,098 & $1: 400$ & $5 \mu \mathrm{g} / \mathrm{mL}$ \\
\hline epifluorescence & goat & chicken & Alexa Fluor 647 & Invitrogen & A21449 & $1: 300$ & $6.7 \mu \mathrm{g} / \mathrm{mL}$ \\
\hline
\end{tabular}


the live histogram of the acquired image.

\subsection{Storm}

For STORM, we use a commercial glucose oxidase-based STORM buffer that allows for robust and reproducible performance (Abbelight Smart Kit dSTORM buffer). Alternatively, we prepare a home-made glucose-oxidase glucose oxidase/catalase/glucose oxygen capture system together with cysteamine (MEA) as a reducing agent:

\subsubsection{Storm buffer}

- $50 \mathrm{~mm}$ Tris pH8, $10 \mathrm{~mm} \mathrm{NaCl}$ (from $2 \mathrm{X}$ stock stored at $4{ }^{\circ} \mathrm{C}$ )

- $10 \%$ glucose (from $25 \%$ stock stored at $4{ }^{\circ} \mathrm{C}$, ThermoFisher, ref. 15023-021).

- $50 \mathrm{~mm}$ MEA (from $1 \mathrm{M}$ stock in $0.36 \mathrm{M} \mathrm{HCl} \mathrm{Sigma,} \mathrm{ref.} \mathrm{60-23-1).} \mathrm{We}$ prepare aliquots of solid MEA corresponding to $\sim 1$ ML of $1 \mathrm{M}$ solution and store them at $-20^{\circ} \mathrm{C}$ in a desiccator. $1 \mathrm{M}$ solution is prepared extemporaneously and kept at $4{ }^{\circ} \mathrm{C}$ for up to 1 week.

- $0.5 \mathrm{Mg} / \mathrm{mL}$ glucose oxidase (Sigma, ref. G2133, from $20 \mathrm{Mg} / \mathrm{mL}$ stock in 50/50 GOD buffer/glycerol stored at $-20^{\circ} \mathrm{C}$. GOD buffer is $24 \mathrm{~mm}$ PIPES, $4 \mathrm{~mm} \mathrm{MgCl}_{2}$, 2 mm EGTA, $\mathrm{pH}$ 6.8).

- $40 \mu \mathrm{g} / \mathrm{mL}$ catalase (Sigma, ref. C40, from $5 \mathrm{mg} / \mathrm{mL}$ stock in 50/50 GOD buffer/glycerol stored at $-20^{\circ} \mathrm{C}$ ).

As this buffer will progressively acidify when in contact with ambient oxygen $[73,74]$, we use a closed chamber made with adhesive silicone inserts (CoverWell chambers, EMS Diasum \#70334-A) attached to glass slide (Fig. 4B). The round $18 \mathrm{~mm}$ coverslip is simply pressed on the chamber overfilled with STORM buffer $(\sim 160 \mu \mathrm{L})$, and suction between glass and silicone allows for good stability, even during long acquisitions. We have not monitored the $\mathrm{pH}$ evolution of our buffer during imaging, but blinking performance is usually stable for up to $4 \mathrm{~h}$ in this sealed silicone chamber. Collapse of buffer performance is usually detected as a slowing down of blinking (longer blinking events) and an inability to drive enough fluorophores in the dark state (appearance of constant fluorescence). Coverslips can be easily mounted and unmounted from the silicone insert, allowing to reuse them over several imaging sessions, and we keep them in phosphate buffer, $0.02 \%$ sodium azide in sealed multi-well plates between sessions.

Once mounted, a suitable cell is located using epifluorescence, then the illumination is switched to the $647 \mathrm{~nm}$ laser at the critical angle incidence and maximum laser power. After a few seconds, most fluorophores are brought to a dark state and the live image shows fast blinking of the activated fluorophores. We then launch the acquisition of 40,000-100,000 frames with an exposure time of 15 Ms $(20,000$ frames/50 Ms on the Abbelight setup), which results in a total acquisition time of 10-20 Min. During acquisition, illumination with the 405 $\mathrm{nm}$ laser is started and progressively raised in order to compensate for the bleaching of fast-blinking fluorophores, and to promote the blinking of fluorophores previously brought to a long-lived dark state. A feedback procedure coupled to live processing of blinking localizations automatically adjust the $405 \mathrm{~nm}$ laser intensity to maintain a constant number of detected localizations per frame. For best results, the goal is to maximize the number of localizations obtained in the minimum amount of time. The limit for this is the density of blinking fluorophores that will allow proper localization by the fitting algorithm [75]. Depending on the intrinsic density of the labeled structure, the $405 \mathrm{~nm}$ activation can be adjusted to stabilize the blinking close to the optimal density during the acquisition. It is thus possible to obtain several millions of localizations within $15 \mathrm{~min}$, delineating cellular structures such as microtubules and clathrin-coated pits with great detail (Fig. 4A and $\mathrm{C}$ ).

Actin labeling using phalloidin is usually the most densely blinking sample, often too dense to properly fit single blinking events. In order to decrease the blinking intensity, we raise the MEA concentration to 100 $\mathrm{mm}$ in the STORM buffer. As mentioned above, stained coverslips are kept in $500 \mathrm{~nm}$ phalloidin-AF647 until imaging. After equilibration at room temperature and three quick rinses with $\mathrm{TpO}_{4}$, coverslips are mounted in STORM buffer for imaging. Moreover, we supplement the STORM buffer with a lower concentration of phalloidin-AF647 $(50 \mathrm{~nm})$ that mitigates the detachment of phalloidin-AF647 during the imaging session, and renews the fluorescent phalloidin on the sample (Fig. 4D) [37].

\section{3. $3 D$ imaging}

3D imaging is performed by inserting a cylindrical lens in the optical path in front of the camera. This results in the deformation of the pointspread function (PSF) into an ellipse of opposite orientation below and above the focal plane [76,77]. After calibration using beads deposited on a coverslip, the shape of the PSF ellipse can be used to fit the position of the blinking fluorophore in $\mathrm{Z}$ over a $\sim 800 \mathrm{~nm}$ range, with a precision 2-3X worse than the lateral localization precision, (s.d. $\sim 20 \mathrm{~nm}, \mathrm{FWHM}$ $50 \mathrm{~nm}$ ) [77]. Due to the enlarged PSF and the added dimension, algorithm for 3D SMLM usually have optimal performance at densities below those attainable in 2D SMLM [78], so this has to be taken into account during acquisition. 3D STORM images can be represented as $\mathrm{Z}$ color-coded images or transverse $\mathrm{XZ} / \mathrm{YZ}$ sections showing the $3 \mathrm{D}$ organization of structures such as microtubules, clathrin-coated pits and actin filaments (Fig. 5).

\subsection{DNA-PAINt}

DNA-PAINT principle is entirely distinct from STORM, but the resulting acquired sequences are similar, with a series of images showing blinking fluorophores. Acquisition of DNA-PAINT images is simpler than STORM, thanks to the separation of the blinking mechanism from the photophysics of the probe and illumination parameters. For each channel to image, the fluorescent imager strand complementary to the docking strand (coupled to the desired secondary antibody) is added to a saline buffer (PBS with $500 \mathrm{~mm} \mathrm{NaCl}, \mathrm{pH}$ 7.2), and imaging is done with continuous illumination at the critical angle. Blinking events density can be easily adjusted by changing the concentration of the imager strand - we typically use $0.1-1 \mathrm{~nm}$. Once set, this density is usually constant over the whole acquisition, although sometimes highpower illumination can photolyze binding sites during long acquisition, resulting in a decreasing blinking event density [79].

One thing to keep in mind is that DNA-PAINT acquisition is slower than STORM. The transient interaction between the 9-basepair docking strand and imager strand can last up to several hundreds of milliseconds, so blinking is significantly slower than in STORM. Typical exposure times of PAINT images are in the range of 100-500 Ms, with the accumulation of tens of thousands of frames taking up to a few hours for a single image $[25,80]$. One way to alleviate this is to use FRET-PAINT $[81,82]$ that allows to raise the imager concentration without obtaining an overwhelming background fluorescence. We are using a simpler method when imaging densely stained targets: raising the laser power to $60-70 \%$ in the PAINT imaging buffer results in faster bleaching of bound probes, shortening each blinking event and decreasing the overall blinking density. This allows for high density blinking at faster frame rates (30-50 Ms exposure time). As the fluorophore bound to the imager strand is usually bleached before it detaches from the docking strand, this does not result in a significant loss of photons emitted or localization precision. We typically acquire sequences of 20,000-40,000 frames for PAINT images.

We use PAINT rather than STORM for multicolor imaging, as we found it difficult to get reliably good blinking using a second fluorophore spectrally distinct from Alexa Fluor 647 [21]. For two-color PAINT, there are two possibilities on our setup. The first is to use two different fluorophores (Atto565 and Atto655) on each imager strand (complementary to the P1 and P3 docking strands), and to image in the 
Table 3

Software tools mentioned in the main text.

\begin{tabular}{|c|c|c|c|}
\hline Name & Reference & Functions & Source/Download \\
\hline 3D-DAOSTORM & Babcock et al., 2012 [84] & localization, processing, rendering & https://github.com/ZhuangLab/storm-analysis \\
\hline Picasso & Schnitzbauer et al., 2017 [46] & localization, processing, rendering & https://github.com/jungmannlab/picasso \\
\hline ThunderSTORM & Ovesny et al., 2014 [86] & localization, processing, rendering & https://github.com/zitmen/thunderstorm \\
\hline pSMLM-3D & Martens et al., 2018 [87] & specific ThunderSTORM version & https://github.com/kjamartens/thunderstorm \\
\hline DoM Utrecht & Chazeau et al., 2016 [88] & localization, processing, rendering & https://github.com/ekatrukha/DoM_Utrecht \\
\hline ZOLA-3D & Aristov et al., 2018 [89] & localization, processing, rendering & https://github.com/imodpasteur/ZOLA-3D \\
\hline fit3Dspline & Li et al., 2018 [48] & localization, rendering & https://github.com/jries/fit3Dcspline \\
\hline SMAP & Sage et al., 2018 [75] & localization, processing, rendering & https://github.com/jries/SMAP \\
\hline ChriSTORM & Leterrier et al., 2015 [36] & scripts for ThunderSTORM & https://github.com/cleterrier/ChriSTORM \\
\hline VISP & El Beheiry \& Dahan, 2013 [95] & $3 \mathrm{D}$ rendering & website Curie Institute \\
\hline PointCloud loader & Felix Woitzel & $3 \mathrm{D}$ rendering & https://github.com/Flexi23/pointcloud-loader \\
\hline NanoJ-SQUIRREL & Culley et al., 2018 [39] & image quality assesment & https://bitbucket.org/rhenriqueslab/nanoj-squirrel/wiki/Home \\
\hline
\end{tabular}

presence of both imager strands. Excitation is alternated every frame between the 561 and $647 \mathrm{~nm}$ lasers using the 4-band cube, and the two channels are acquired in a single acquisition sequence. One advantage of this procedure is that drift occurs in parallel for both channels and that alignment is easier, although the chromatic aberration between the two spectrally-distinct channels has to be corrected [83]. The other possibility, that we tend to favor because of its lower crosstalk between channels, is to use the same fluorophore for each imager with sequential acquisition separated by washing out of the first imager strand. In this case, we use far-red imagers (Atto655) with the single-emission band cube that result in a better signal (Fig. 6A). Channels must be corrected independently for drift and subsequently aligned using common features in both channels, and the use of the same fluorophore alleviates the need for chromatic correction. Both procedures can be combined for more than two colors: using sequential acquisition with two passes of two imagers (distinct fluorophores), we are able to acquire 4-color images of different targets (Fig. 6B). For easier rinses and medium exchange during these complex sequential imaging protocols, we mount the $18 \mathrm{~mm}$ round coverslips in an open metal chamber (Ludin chamber, Life Imaging Services) for PAINT imaging, and have devised an automatized pump system in LEGO driven by an Arduino computer and open-source software [29].

Finally, PAINT can be sequentially combined with STORM, and this is useful to image actin in addition to antibody-labeled structures. If actin is labeled with phalloidin-AF647, it is imaged first in STORM buffer, before imaging the PAINT channels sequentially using far-red imagers. To avoid residual actin staining interfering with the PAINT images, a $\sim 10$ Min bleaching step of the phalloidin-AF647 in PAINT imaging buffer at maximum $647 \mathrm{~nm}$ laser power must be performed before adding the first far-red PAINT imager. This allows to obtain the best actin images and combine them with other targets such as microtubules and clathrin (Fig. 6A). Alternatively, we use phalloidin-Atto488 that is well separated from the far-red PAINT channel and can blink in regular phosphate buffer or PAINT imaging buffer because of spontaneous quenching of cyanine dyes by the tryptophan residue of phalloidin [84]. STORM of actin is acquired first, with no imager present, before the acquisition of the PAINT channels (Fig. 6B) [29]. There is significant bleaching of the phalloidin-Atto488 over time during the STORM acquisition, and Atto488 is insensitive to $405 \mathrm{~nm}$ activation. This ultimately limits the final quality of the actin image compared to phalloidin-AF647.

\section{Processing}

\subsection{Fitting of the blinking events into localization coordinates}

As SMLM images come from mathematical fitting performed over acquired images, processing is an integral part of the imaging workflow. It is important to have a good grasp of how this processing is performed, in order to be able to optimize parameters and avoid artifacts. The primary and most important processing step is the fitting of the blinking events: each peak on images from the acquisition sequence is detected, then fitted to determine the precise localization of the event. Fitting can be done using different algorithms, the most common ones being gaussian fitting with maximum likelihood or leastsquare fitting $[85,86]$. 3D SMLM involves localization in $\mathrm{Z}$ by fitting the point-spread function shape to a calibration curve. For densely labeled samples, specific algorithms are available that are able to fit overlapping peaks $[78,87,88]$. Detection threshold (to decide what will be fitted) and rejection parameters (to avoid fitting peaks that are not single molecule blinking events) are important and should be adjusted to the acquisition conditions.

SMLM localization algorithms are now quite mature, with several software showing good performance. Rigorous evaluations of performance on standardized benchmark tests have been published for a number of software options both for 2D and 3D-SMLM, providing an unbiased overview of the available options $[78,86]$. We will mention a few that we have tested extensively and found to give qualitatively satisfactory results on our data. We use the Nikon N-STORM localization software that is based on 3D-DAOSTORM and is available as an open-source python software [89]. The Abbelight Neo software is also used to process images acquired with this setup. A strong open-source option is Picasso [48], a Python software that integrates GPU-accelerated least-square fitting [90] with various post-processing options. Several options are available in the open-source ImageJ/Fiji ecosystem [91]: a popular choice is ThunderSTORM [92], in particular the newer version integrating the phasor fitting approach, which is very fast and exists as a Fiji update site [93]. Another good option is DoM (Detection of Molecules) Utrecht, a fast plugin that integrates options such as chromatic aberration correction [94]. A recently-developed alternative is ZOLA-3D, which can fit any PSF shape including the ones used for extended Z-range [95]. There are also MATLAB-based software solutions that have good performance, such as fit3dspline and SMAP $[50,78]$. When properly used with optimized parameters, these software can provide good images from 2D and 3D SMLM acquisitions (Fig. 7, Table 3).

\subsection{Post-processing of localization coordinates}

Once all blinking events have been fitted, several post-processing steps are necessary before reconstructing an image. The first one is drift correction: mechanical and thermal drift over tens to hundreds of nanometers occur during SMLM acquisitions, and proper drift correction is necessary to obtain a good image. For strongly structured images such as the cell cytoskeleton, the method of choice is the cross-correlation of images from parts of the acquisition sequence. Partial images are reconstructed using sub-sequences of $\sim 1000$ frames and the drift is determined by cross-correlation with the preceding sub-sequence reconstruction, then interpolated within each frame of the sub-sequence. The best performance is obtained using redundant cross-correlation, 
where each sub-sequence reconstruction is correlated with all others [96]. It also possible to add fiducials to the sample, such as fluorescent or gold beads and use them to correct drift at the processing stage [15], or to monitor drift during acquisition using brightfield imaging [97]. Drift correction is usually performed in $2 \mathrm{D}$ as a focus stabilization is used to avoid Z drift. However, Z drift can nevertheless occur [98] and can be further corrected by post-processing correction (3D-DAOSTORM, ZOLA-3D). Finally, sequential application of several drift correction steps can lead to progressively better results.

Another important post-processing step is to group together localizations from the same blinking event. Blinking events can sometimes last for two or more frames, and this is particularly true for DNA-PAINT acquisition where interaction between DNA strands can be long-lasting. In order to obtain a more faithful reconstruction with more precise localizations, this processing step groups together localizations that appear on subsequent frames within a given radius and averages the resulting localization (with a precision provided by photons from each frame). We usually set the radius to one camera pixel (160 nm on the NSTORM setup, $97 \mathrm{~nm}$ on the Abbelight setup). It is sometimes possible to specify a number of "dark" frames allowing transient disappearance of the blinking event.

Finally, it is important to filter the resulting localization list to remove localizations that are not good enough: one can filter on the least square fitting $\chi^{2}$ (to exclude bad fits), the number of successive detections for grouped localizations (to avoid long-lasting events that are often artifacts), the number of photons emitted or the localization precision (to exclude blinking events that are too faint). Filtering on the size of the blinking event width can provide virtual optical sectioning by restricting the localizations to those very close to the focal plane [99]. Filtering the few localization events that have a bad precision can also speed up the reconstruction when using Gaussian rendering with individual precision values, as these localizations will contribute a low amount of intensity to a large number of pixels in the final image.

In our workflow, drift correction and localization grouping are done in the N-STORM software. When performing simultaneous two-color DNA-PAINT using red and far-red imagers, correction of the chromatic aberration is performed in the N-STORM software using a polynomial warp fitting calibrated on dense fields of Tetraspeck beads [100]. After translation of the localization coordinates list in the proper commaseparated value (CSV) format, we do further post-processing in ThunderSTORM using custom scripts (ChriSTORM) [38], including another drift correction step and filtering of localizations. For STORM, we usually filter out localization events with less than 800 or more than 50,000 photons, and those lasting more than 5 successive frames. For PAINT, the upper limit is raised to 200,000 for photons emitted and to 50 for the number of successive frames.

\subsection{Image reconstruction}

After fitting and post-processing, the final list of localization coordinates is the primary data format of SMLM. These can be used to reconstruct images, and several methods are available for this [101]: histogram (the image is divided in a pixel grid and each pixel intensity depends on the number of localizations it contains), Gaussian rendering (a Gaussian is drawn at each localization coordinates), Delaunay triangulation (the image is tiled with one localization in each tile, the intensity being inversely proportional to the size of the tile). In the Gaussian case, the size of the Gaussian can be fixed (ideally to the average uncertainty of all localizations) or each can be drawn with its individual localization uncertainty depending on the number of photons emitted by the corresponding blinking event. The Gaussian rendering with individual precision values is more complex to generate but leads to the most precise reconstructions [102]. It is thus important for the fitting software to calculate, or at least approximate, the localization precision for each blinking event in order to use it for reconstruction. Finally, 3D STORM images can be reconstructed and visualized either as color-coded projections, or directly in 3D thanks to specific software such as ViSP [103] or the web-based Pointcloud Loader (Table 3).

\subsection{Analysis of SMLM data}

SMLM can provide fascinating image with extraordinary details of cellular structures. However, the primary data can (and should) be exploited beyond the reconstruction of images, preferably using coordinate-based analysis that directly use the localization list rather than the reconstructed image [104]. This includes clustering and segmentation into structures, quantification of the nanoscale morphology, or relationships between distinct structures [105-107]. At this scale, colocalization does not exist and is replaced by the measurement of distances between the localizations of different species [108-110]. A recent development in SMLM analysis is the use of single particle averaging to reach molecular details beyond image resolution by averaging multiple similar objects [111-115]. A detailed review of the available algorithms and software is beyond the scope of the present article, but we encourage the interested reader to explore this very dynamic area that constitutes the next logical step toward leveraging SMLM possibilities. As an example, recent tools have been developed to assess the resolution and quality of SMLM images, allowing users to rapidly detect problems and optimize their imaging. Resolution in SMLM is tricky to define, as it involves not only the localization precision, but also the labeling density necessary to properly sample the structure of interest. Localization precision can be estimated over a single acquisition sequence by using localizations appearing on successive frames [116], and Fourier ring correlation (FRC), an approach derived from single-particle electron microscopy, can provide resolution values that takes both aspects into account $[117,118]$. Beyond resolution numbers, image quality can be assessed to detect artifacts and optimize any step of the SMLM workflow thanks to NanoJ-SQUIRREL, based on the comparison of the super-resolved image and its diffraction-limited counterpart [41].

\section{Conclusion}

The exquisite resolution and access to single molecule measurements makes SMLM an attractive approach among the various superresolution methods. It has brought crucial insights into the nanoscale organization of cells $[6,119]$. Commercial SMLM systems have been available for ten years, and they can be found in a lot of imaging facilities. However, SMLM is a technique that is still often considered as "difficult": indeed, it requires careful planning and realization of the experiments, and each image takes time to acquire, in particular when performing multi-color PAINT experiments. In our experience, sample preparation is the most crucial point for good SMLM results and optimizing sample preservation while optimizing labeling density usually requires time and efforts. Processing principles must also be understood, in order to avoid the fabrication of artifacts [120]. Finally, taking advantage of single molecule data to obtain meaningful morphological and molecular insight is a current challenge, with a very active community developing new algorithms and software [104]. We hope that our detailed workflow can serve as a good starting point and our advice help as pointers for further specific optimization.

\section{CRediT authorship contribution statement}

Angélique Jimenez: Formal analysis, Investigation, Methodology, Writing - review \& editing. Karoline Friedl: Formal analysis, Investigation, Methodology, Writing - review \& editing. Christophe Leterrier: Conceptualization, Formal analysis, Funding acquisition, Investigation, Methodology, Visualization, Software, Supervision, Writing - original draft, Writing - review \& editing. 


\section{Acknowledgements}

We thank Ghislaine Caillol, Fanny Boroni-Rueda, Sofia Yousfi and Martin Babeau for their help in preparing samples. We thank Pedro Pereira and Ricardo Henriques for DNA-PAINT secondary antibodies and help in preparing them.

\section{Funding}

This work is supported by a grant to Christophe Leterrier from CNRS (ATIP AO2016).

\section{Competing interests}

Karoline Friedl is an employee of Abbelight.

\section{References}

[1] E. Hecht, Optics, Addison-Wesley, 2015.

[2] J. Vangindertael, R. Camacho, W. Sempels, H. Mizuno, P. Dedecker, K.P.F. Janssen, An introduction to optical super-resolution microscopy for the adventurous biologist, Methods Appl. Fluoresc. 6 (2018) 022003, , https://doi. org/10.1088/2050-6120/aaae0c.

[3] R. Milo, R. Phillips, Cell biology by the numbers, Garland, Science (2015).

[4] L. Schermelleh, A. Ferrand, T. Huser, C. Eggeling, M. Sauer, O. Biehlmaier, et al., Super-resolution microscopy demystified, Nat. Cell Biol. 21 (2019) 72-84, https:// doi.org/10.1038/s41556-018-0251-8.

[5] S.J. Sahl, S.W. Hell, S. Jakobs, Fluorescence nanoscopy in cell biology, Nat. Rev. Mol. Cell Biol. 9 (2017) 1-17, https://doi.org/10.1038/nrm.2017.71.

[6] Y.M. Sigal, R. Zhou, X. Zhuang, Visualizing and discovering cellular structures with super-resolution microscopy, Science 361 (2018) 880-887, https://doi.org/ 10.1126/science.aau1044.

[7] C. Sieben, K.M. Douglass, P. Guichard, S. Manley, Super-resolution microscopy to decipher multi-molecular assemblies, Curr. Opin. Struct. Biol. 49 (2018) 169-176, https://doi.org/10.1016/j.sbi.2018.03.017.

[8] M. Bachmann, F. Fiederling, M. Bastmeyer, Practical limitations of superresolution imaging due to conventional sample preparation revealed by a direct comparison of CLSM, SIM and dSTORM, J. Microsc. 262 (2016) 306-315, https://doi.org/10. 1111/jmi.12365.

[9] E. Wegel, A. Göhler, B.C. Lagerholm, A. Wainman, S. Uphoff, R. Kaufmann, et al., Imaging cellular structures in super-resolution with SIM STED and Localisation Microscopy: a practical comparison, Sci Rep. 6 (2016) 27290, https://doi.org/10. 1038/srep27290.

[10] R.E. Thompson, D.R. Larson, W.W. Webb, Precise nanometer localization analysis for individual fluorescent probes, Biophys. J . 82 (2002) 2775-2783, https://doi. org/10.1016/S0006-3495(02)75618-X.

[11] K.I. Mortensen, L.S. Churchman, J.A. Spudich, H. Flyvbjerg, Optimized localization analysis for single-molecule tracking and super-resolution microscopy, Nat. Methods 7 (2010) 377-381, https://doi.org/10.1038/nmeth.1447.

[12] T. Schmidt, G.J. Schütz, W. Baumgartner, H.J. Gruber, H. Schindler, Imaging of single molecule diffusion, PNAS 93 (1996) 2926-2929, https://doi.org/10.1021/ acs.jpcb.8b08980.

[13] A. Yildiz, Myosin V Walks, Hand-over-hand: single fluorophore imaging with 1.5nm localization, Science 300 (2003) 2061-2065, https://doi.org/10.1126/ science. 1084398

[14] E. Betzig, Proposed method for molecular optical imaging, Opt. Lett. 20 (1995) 237-239.

[15] E. Betzig, G.H. Patterson, R. Sougrat, O.W. Lindwasser, S. Olenych, J.S. Bonifacino, et al., Imaging intracellular fluorescent proteins at nanometer resolution, Science 313 (2006) 1642-1645, https://doi.org/10.1126/science. 1127344.

[16] S.T. Hess, T.P.K. Girirajan, M.D. Mason, Ultra-high resolution imaging by fluorescence photoactivation localization microscopy, Biophys. J . 91 (2006) 4258-4272, https://doi.org/10.1529/biophysj.106.091116.

[17] S. Manley, J.M. Gillette, G.H. Patterson, H. Shroff, H.F. Hess, E. Betzig, et al., Highdensity mapping of single-molecule trajectories with photoactivated localization microscopy, Nat. Methods 5 (2008) 155-157, https://doi.org/10.1038/nmeth. 1176.

[18] M.J. Rust, M. Bates, X. Zhuang, Sub-diffraction-limit imaging by stochastic optical reconstruction microscopy (STORM), Nat. Methods 3 (2006) 793-795, https:// doi.org/10.1038/nmeth929.

[19] M. Heilemann, S. van de Linde, M. Schüttpelz, R. Kasper, B. Seefeldt, A. Mukherjee, et al., Subdiffraction-resolution fluorescence imaging with conventional fluorescent probes, Angew. Chem. Int. Ed. Engl. 47 (2008) 6172-6176, https://doi.org/10.1002/anie.200802376.

[20] A.R. Halpern, M.D. Howard, J.C. Vaughan, Point by point: an introductory guide to sample preparation for single-molecule, super-resolution fluorescence microscopy, Curr. Protoc. Chem. Biol. 7 (2015) 103-120, https://doi.org/10.1002/ 9780470559277.ch140241.

[21] G.T. Dempsey, J.C. Vaughan, K.H. Chen, M. Bates, X. Zhuang, Evaluation of fluorophores for optimal performance in localization-based super-resolution imaging, Nat. Methods 8 (2011) 1027-1036, https://doi.org/10.1038/nmeth.1768.

[22] M. Lehmann, G. Lichtner, H. Klenz, J. Schmoranzer, Novel organic dyes for multicolor localization-based super-resolution microscopy, J. Biophotonics 9 (2016) 161-170, https://doi.org/10.1002/jbio.201500119.

[23] A. Sharonov, R.M. Hochstrasser, Wide-field subdiffraction imaging by accumulated binding of diffusing probes, PNAS 103 (2006) 18911-18916, https://doi. org/10.1073/pnas.0609643104.

[24] J. Molle, M. Raab, S. Holzmeister, D. Schmitt-Monreal, D. Grohmann, Z. He, et al., Superresolution microscopy with transient binding, Curr. Opin. Biotechnol. 39 (2016) 8-16, https://doi.org/10.1016/j.copbio.2015.12.009.

[25] R. Jungmann, M.S. Avendaño, J.B. Woehrstein, M. Dai, W.M. Shih, P. Yin, Multiplexed 3D cellular super-resolution imaging with DNA-PAINT and ExchangePAINT, Nat. Methods 11 (2014) 313-318, https://doi.org/10.1038/nmeth.2835.

[26] D.J. Nieves, K. Gaus, M.A.B. Baker, DNA-based super-resolution microscopy: DNAPAINT, Genes (Basel) 9 (2018), https://doi.org/10.3390/genes9120621.

[27] Y. Wang, J.B. Woehrstein, N. Donoghue, M. Dai, M.S. Avendaño, R.C.J. Schackmann, et al., Rapid sequential in situ multiplexing with DNA exchange imaging in neuronal cells and tissues, Nano Lett. 17 (2017) 6131-6139, https://doi.org/10.1021/acs.nanolett.7b02716.

[28] S.S. Agasti, Y. Wang, F. Schueder, A. Sukumar, R. Jungmann, P. Yin, DNA-barcoded labeling probes for highly multiplexed Exchange-PAINT imaging, Chem. Sci. (2017), https://doi.org/10.1039/C6SC05420J.

[29] P. Almada, P.M. Pereira, S. Culley, G. Caillol, F. Boroni-Rueda, C.L. Dix, et al., Automating multimodal microscopy with NanoJ-Fluidics, Nat. Commun. 10 (2019) 1223, https://doi.org/10.1038/s41467-019-09231-9.

[30] J.L. Werbin, M.S. Avendaño, V. Becker, R. Jungmann, P. Yin, G. Danuser, et al., Multiplexed exchange-PAINT imaging reveals ligand-dependent EGFR and Met interactions in the plasma membrane, Sci. Rep. 7 (2017) 12150, https://doi.org/ 10.1038/s41598-017-12257-y.

[31] D. Baddeley, J. Bewersdorf, Biological insight from super-resolution microscopy: what we can learn from localization-based images, Annu. Rev. Biochem. 87 (2018) 965-989, https://doi.org/10.1146/annurev-biochem-060815-014801.

[32] Z. Liu, L.D. Lavis, E. Betzig, Imaging live-cell dynamics and structure at the singlemolecule level, Mol. Cell 58 (2015) 644-659, https://doi.org/10.1016/j.molcel. 2015.02.033.

[33] T.J. Lambert, J.C. Waters, Navigating challenges in the application of superresolution microscopy, J. Cell Biol. 216 (2017) 53-63, https://doi.org/10.1083/ jcb.201610011.

[34] C. Leterrier, P. Dubey, S. Roy, The nano-architecture of the axonal cytoskeleton, Nat. Rev. Neurosci. 18 (2017) 713-726, https://doi.org/10.1038/nrn.2017.129.

[35] M.-J. Papandréou, C. Leterrier, The functional architecture of axonal actin, Mol. Cell. Neurosci. 91 (2018) 151-159, https://doi.org/10.1016/j.mcn.2018.05.003.

[36] C.Y.-M. Huang, C. Zhang, T.S.-Y. Ho, J. Oses-Prieto, A.L. Burlingame, J. Lalonde, et al., $\alpha$ II spectrin forms a periodic cytoskeleton at the axon initial segment and is required for nervous system function, J. Neurosci. 37 (2017) 11311-11322, https://doi.org/10.1523/JNEUROSCI.2112-17.2017.

[37] A. Ganguly, Y. Tang, L. Wang, K. Ladt, J. Loi, B. Dargent, et al., A dynamic formindependent deep F-actin network in axons, J. Cell Biol. 104 (2015) 20576-21417, https://doi.org/10.1083/jcb.201506110.

[38] C. Leterrier, J. Potier, G. Caillol, C. Debarnot, F. Rueda-Boroni, B. Dargent, Nanoscale architecture of the axon initial segment reveals an organized and robust scaffold, Cell Rep. 13 (2015) 2781-2793, https://doi.org/10.1016/j.celrep.2015. 11.051.

[39] A. Ganguly, X. Han, U. Das, L. Wang, J. Loi, J. Sun, et al., Hsc70 chaperone activity is required for the cytosolic slow axonal transport of synapsin, J. Cell Biol. 216 (2017) 2059-2074, https://doi.org/10.1083/jcb.201604028.

[40] N. Chakrabarty, P. Dubey, Y. Tang, A. Ganguly, K. Ladt, C. Leterrier, et al., Processive flow by biased polymerization mediates the slow axonal transport of actin, J. Cell Biol. 218 (2019) 112-124, https://doi.org/10.1083/jcb.201711022.

[41] S. Culley, D. Albrecht, C. Jacobs, P.M. Pereira, C. Leterrier, J. Mercer, et al., Quantitative mapping and minimization of super-resolution optical imaging artifacts, Nat. Methods 15 (2018) 263-266, https://doi.org/10.1038/nmeth.4605.

[42] R.F. Laine, K.L. Tosheva, N. Gustafsson, R.D.M. Gray, P. Almada, D. Albrecht, et al., NanoJ: a high-performance open-source super-resolution microscopy toolbox, J. Phys. D Appl. Phys. (2019), https://iopscience.iop.org/article/10. 1088/1361-6463/ab0261.

[43] P. Hoess, M. Mund, M. Reitberger, J. Ries, Dual-color and 3D super-resolution microscopy of multi-protein assemblies, Methods Mol. Biol. 1764 (2018) 237-251, https://doi.org/10.1007/978-1-4939-7759-8_14.

[44] S. van de Linde, A. Löschberger, T. Klein, M. Heidbreder, S. Wolter, M. Heilemann, et al., Direct stochastic optical reconstruction microscopy with standard fluorescent probes, Nat. Protoc. 6 (2011) 991-1009, https://doi.org/10.1038/nprot. 2011.336.

[45] J.R. Allen, S.T. Ross, M.W. Davidson, Sample preparation for single molecule localization microscopy, PCCP 15 (2013) 18771-18783, https://doi.org/10.1039/ c3cp53719f.

[46] P.M. Pereira, P. Almada, R. Henriques, High-content 3D multicolor super-resolution localization microscopy, Methods Cell Biol. 125 (2015) 95-117, https://doi. org/10.1016/bs.mcb.2014.10.004.

[47] J. Xu, H. Ma, Y. Liu, Stochastic Optical Reconstruction Microscopy (STORM), Curr Protoc Cytom. 81 (2017) 12.46.1-12.46.27, https://doi.org/10.1002/cpcy.23.

[48] J. Schnitzbauer, M.T. Strauss, T. Schlichthaerle, F. Schueder, R. Jungmann, Superresolution microscopy with DNA-PAINT, Nat. Protoc. 12 (2017) 1198-1228, https://doi.org/10.1038/nprot.2017.024.

[49] Y. Gluzman, SV40-transformed simian cells support the replication of early SV40 
mutants, Cell 23 (1981) 175-182.

[50] Y. Li, M. Mund, P. Hoess, J. Deschamps, U. Matti, B. Nijmeijer, et al., Real-time 3D single-molecule localization using experimental point spread functions, Nat. Methods 15 (2018) 367-369, https://doi.org/10.1038/nmeth.4661.

[51] M. Bates, B. Huang, G.T. Dempsey, X. Zhuang, Multicolor super-resolution imaging with photo-switchable fluorescent probes, Science 317 (2007) 1749-1753, https://doi.org/10.1126/science.1146598.

[52] D. Lin, L.A. Gagnon, M.D. Howard, A.R. Halpern, J.C. Vaughan, Extended-depth 3D super-resolution imaging using probe-refresh STORM, Biophys. J . 114 (2018) 1980-1987, https://doi.org/10.1016/j.bpj.2018.03.023.

[53] M. Gao, R. Maraspini, O. Beutel, A. Zehtabian, B. Eickholt, A. Honigmann, et al., Expansion stimulated emission depletion microscopy (ExSTED), ACS Nano (2018), https://doi.org/10.1021/acsnano.8b00776.

[54] R.J. Geraghty, A. Capes-Davis, J.M. Davis, J. Downward, R.I. Freshney, I. Knezevic, et al., Guidelines for the use of cell lines in biomedical research, Br. J. Cancer 111 (2014) 1021-1046, https://doi.org/10.1038/bjc. 2014.166.

[55] S. Kaech, G.A. Banker, Culturing hippocampal neurons, Nat. Protoc. 1 (2006) 2406-2415.

[56] D.R. Whelan, T.D.M. Bell, Image artifacts in Single Molecule Localization Microscopy: why optimization of sample preparation protocols matters, Sci. Rep. 5 (2015) 7924, https://doi.org/10.1038/srep07924.

[57] J.C. Bulinski, Immunofluorescence localization of HeLa cell microtubule-associated proteins on microtubules in vitro and in vivo, J. Cell Biol. 87 (1980) 792-801, https://doi.org/10.1083/jcb.87.3.792.

[58] D. Leyton-Puig, K.M. Kedziora, T. Isogai, B. van den Broek, K. Jalink, M. Innocenti, PFA fixation enables artifact-free super-resolution imaging of the actin cytoskeleton and associated proteins, Biology Open. 5 (2016) 1001-1009, https://doi. org/10.1242/bio.019570.

[59] Y.M. Sigal, C.M. Speer, H.P. Babcock, X. Zhuang, Mapping synaptic input fields of neurons with super-resolution imaging, Cell 163 (2015) 493-505, https://doi.org/ 10.1016/j.cell.2015.08.033.

[60] M. Mikhaylova, B.M.C. Cloin, K. Finan, R. van den Berg, J. Teeuw, M.M. Kijanka, et al., Resolving bundled microtubules using anti-tubulin nanobodies, Nat. Commun. 6 (2015) 7933, https://doi.org/10.1038/ncomms8933.

[61] T. Pleiner, M. Bates, D. Görlich, A toolbox of anti-mouse and anti-rabbit IgG secondary nanobodies, J. Cell Biol. 217 (2018) 1143-1154, https://doi.org/10. 1083/jcb. 201709115

[62] A. Dani, B. Huang, J. Bergan, C. Dulac, X. Zhuang, Superresolution imaging of chemical synapses in the brain, Neuron 68 (2010) 843-856, https://doi.org/10 1016/j.neuron.2010.11.021.

[63] K. Xu, H.P. Babcock, X. Zhuang, Dual-objective STORM reveals three-dimensional filament organization in the actin cytoskeleton, Nat. Methods 9 (2012) 185-188, https://doi.org/10.1038/nmeth.1841.

[64] T. Schlichthaerle, A.S. Eklund, F. Schueder, M.T. Strauss, C. Tiede, A. Curd, et al., Site-specific labeling of affimers for DNA-PAINT microscopy, Angew. Chem. Int. Ed. Engl. 57 (2018) 11060-11063, https://doi.org/10.1002/anie.201804020.

[65] T. Kiuchi, M. Higuchi, A. Takamura, M. Maruoka, N. Watanabe, Multitarget superresolution microscopy with high-density labeling by exchangeable probes, Nat. Methods (2015), https://doi.org/10.1038/nmeth.3466.

[66] G.W. Ashdown, G.L. Burn, D.J. Williamson, E. Pandzic, R. Peters, M. Holden, et al., Live-cell super-resolution reveals F-actin and plasma membrane dynamics at the $\mathrm{T}$ cell synapse, Biophys. J . 112 (2017) 1703-1713, https://doi.org/10.1016/j.bpj 2017.01.038.

[67] R.P. Tas, T.G.A.A. Bos, L.C. Kapitein, Purification and application of a small actin probe for single-molecule localization microscopy, Methods Mol. Biol. 1665 (2018) 155-171, https://doi.org/10.1007/978-1-4939-7271-5_9.

[68] T. Nakata, N. Hirokawa, Microtubules provide directional cues for polarized axonal transport through interaction with kinesin motor head, J. Cell Biol. 162 (2003) 1045-1055, https://doi.org/10.1083/jcb.200302175.

[69] J. Sinkó, G. Szabó, M. Erdélyi, Ray tracing analysis of inclined illumination techniques, Opt. Express (2014), https://doi.org/10.1364/OE.22.018940.

[70] M. Tokunaga, N. Imamoto, K. Sakata-Sogawa, Highly inclined thin illumination enables clear single-molecule imaging in cells, Nat. Methods 5 (2008) 159-161, https://doi.org/10.1038/nmeth1171.

[71] Y. Guo, D. Li, S. Zhang, Y. Yang, J.-J. Liu, X. Wang, et al., Visualizing intracellular organelle and cytoskeletal interactions at nanoscale resolution on millisecond timescales, Cell (2018), https://doi.org/10.1016/j.cell.2018.09.057.

[72] C.K. Carniglia, L. Mandel, K.H. Drexhage, Absorption and emission of evanescent photons, J. Opt. Soc. Am. 62 (1972) 479, https://doi.org/10.1364/JOSA.62. 000479.

[73] N. Olivier, D. Keller, P. Gönczy, S. Manley, Resolution doubling in 3D-STORM imaging through improved buffers, PLoS ONE 8 (2013) e69004, , https://doi.org/ 10.1371/journal.pone.0069004.

[74] M. Swoboda, J. Henig, H.-M. Cheng, D. Brugger, D. Haltrich, N. Plumeré, et al., Enzymatic oxygen scavenging for photostability without $\mathrm{pH}$ drop in single-molecule experiments, ACS Nano 6 (2012) 6364-6369, https://doi.org/10.1021/ nn301895c.

[75] S. Wolter, U. Endesfelder, S. van de Linde, M. Heilemann, M. Sauer, Measuring localization performance of super-resolution algorithms on very active samples, Opt. Express 19 (2011) 7020-7033, https://doi.org/10.1364/OE.19.007020.

[76] H.P. Kao, A.S. Verkman, Tracking of single fluorescent particles in three dimensions: use of cylindrical optics to encode particle position, Biophys. J . 67 (1994) 1291-1300, https://doi.org/10.1016/S0006-3495(94)80601-0.

[77] B. Huang, W. Wang, M. Bates, X. Zhuang, Three-dimensional super-resolution imaging by stochastic optical reconstruction microscopy, Science 319 (2008) 810-813, https://doi.org/10.1126/science.1153529.
[78] D. Sage, T.-A. Pham, H.P. Babcock, T. Lukes, T. Pengo, J. Chao, et al., Superresolution fight club: assessment of 2D and 3D single-molecule localization microscopy software, Nat. Methods 313 (2019) 1642, https://doi.org/10.1038/ s41592-019-0364-4.

[79] P. Blumhardt, J. Stein, J. Mücksch, F. Stehr, J. Bauer, R. Jungmann, et al., Photoinduced depletion of binding sites in DNA-PAINT microscopy, Molecules 23 (2018), https://doi.org/10.3390/molecules23123165.

[80] T. Schlichthaerle, M. Ganji, A. Auer, O.K. Wade, R. Jungmann, Bacterial-derived antibody binders as small adapters for DNA-PAINT microscopy, ChemBioChem (2018), https://doi.org/10.1002/cbic.201800743.

[81] A. Auer, M.T. Strauss, T. Schlichthaerle, R. Jungmann, Fast background-free DNAPAINT imaging using FRET-based probes, Nano Lett. 17 (2017) 6428-6434, https://doi.org/10.1021/acs.nanolett.7b03425.

[82] J. Lee, S. Park, W. Kang, S. Hohng, Accelerated super-resolution imaging with FRET-PAINT, Mol Brain 10 (2017) 63, https://doi.org/10.1186/s13041-0170344-5.

[83] M. Erdelyi, E. Rees, D. Metcalf, G.S.K. Schierle, L. Dudas, J. Sinko, et al., Correcting chromatic offset in multicolor super-resolution localization microscopy, Opt. Express 21 (2013) 10978-10988, https://doi.org/10.1364/OE.21. 010978.

[84] S. Nanguneri, B. Flottmann, F. Herrmannsdörfer, T. Kuner, M. Heilemann, Singlemolecule super-resolution imaging by tryptophan-quenching-induced photoswitching of phalloidin-fluorophore conjugates, Microsc. Res. Tech. 77 (2014) 510-516, https://doi.org/10.1002/jemt.22349.

[85] H. Deschout, F.C. Zanacchi, M. Mlodzianoski, A. Diaspro, J. Bewersdorf, S.T. Hess, et al., Precisely and accurately localizing single emitters in fluorescence microscopy, Nat. Methods 11 (2014) 253-266, https://doi.org/10.1038/nmeth.2843.

[86] D. Sage, H. Kirshner, T. Pengo, N. Stuurman, J. Min, S. Manley, et al., Quantitative evaluation of software packages for single-molecule localization microscopy, Nat. Methods 12 (2015) 717-724, https://doi.org/10.1038/nmeth.3442.

[87] S.J. Holden, S. Uphoff, A.N. Kapanidis, DAOSTORM: an algorithm for high- density super-resolution microscopy, Nat. Methods 8 (2011) 279-280, https://doi. org/10.1038/nmeth0411-279.

[88] S. Mailfert, J. Touvier, L. Benyoussef, R. Fabre, A. Rabaoui, M.-C. Blache, et al., A theoretical high-density nanoscopy study leads to the design of UNLOC, a parameter-free algorithm, Biophys. J. 115 (2018) 565-576, https://doi.org/10.1016/j. bpj.2018.06.024.

[89] H.P. Babcock, Y.M. Sigal, X. Zhuang, A high-density 3D localization algorithm for stochastic optical reconstruction microscopy, Opt. Nanosc. 1 (2012) 6, https://doi. org/10.1186/2192-2853-1-6.

[90] A. Przybylski, B. Thiel, J. Keller-Findeisen, B. Stock, M. Bates, Gpufit: an opensource toolkit for GPU-accelerated curve fitting, Sci. Rep. 7 (2017) 15722, https:// doi.org/10.1038/s41598-017-15313-9.

[91] S. van de Linde, Single-molecule localization microscopy analysis with ImageJ, J. Phys. D Appl. Phys. 52 (2019) 203002, , https://doi.org/10.1088/1361-6463/ ab092f.

[92] M. Ovesny, P. Křižek, J. Borkovec, Z. Svindrych, G.M. Hagen, ThunderSTORM: a comprehensive ImageJ plug-in for PALM and STORM data analysis and superresolution imaging, Bioinformatics 30 (2014) 2389-2390, https://doi.org/10. 1093/bioinformatics/btu202.

[93] K.J.A. Martens, A.N. Bader, S. Baas, B. Rieger, J. Hohlbein, Phasor based singlemolecule localization microscopy in 3D (pSMLM-3D): an algorithm for $\mathrm{MHz}$ localization rates using standard CPUs, J. Chem. Phys. 148 (2018) 123311, , https:// doi.org/10.1063/1.5005899.

[94] A. Chazeau, E.A. Katrukha, C.C. Hoogenraad, L.C. Kapitein, Studying neuronal microtubule organization and microtubule-associated proteins using single molecule localization microscopy, Methods Cell Biol. 131 (2016) 127-149, https://doi. org/10.1016/bs.mcb.2015.06.017.

[95] A. Aristov, B. Lelandais, E. Rensen, C. Zimmer, ZOLA-3D allows flexible 3D localization microscopy over an adjustable axial range, Nat. Commun. 9 (2018) 1642, https://doi.org/10.1038/s41467-018-04709-4.

[96] Y. Wang, J. Schnitzbauer, Z. Hu, X. Li, Y. Cheng, Z.-L. Huang, et al., Localization events-based sample drift correction for localization microscopy with redundant cross-correlation algorithm, Opt. Express 22 (2014) 15982-15991, https://doi. org/10.1364/OE.22.015982.

[97] R. McGorty, D. Kamiyama, B. Huang, Active microscope stabilization in three dimensions using image correlation, Opt Nanosc. 2 (2013) 3, https://doi.org/10. 1186/2192-2853-2-3.

[98] C. Cabriel, N. Bourg, P. Jouchet, G. Dupuis, C. Leterrier, A. Baron, et al., Combining 3D single molecule localization strategies for reproducible bioimaging, Nat. Commun. 10 (2019) 1642, https://doi.org/10.1038/s41467-019-09901-8.

[99] M. Palayret, H. Armes, S. Basu, A.T. Watson, A. Herbert, D. Lando, et al., Virtual"light-sheet" single-molecule localisation microscopy enables quantitative optical sectioning for super-resolution imaging, PLoS ONE 10 (2015) e0125438, , https:// doi.org/10.1371/journal.pone.0125438.

[100] M. Bates, G.T. Dempsey, K.H. Chen, X. Zhuang, Multicolor super-resolution fluorescence imaging via multi-parameter fluorophore detection, ChemPhysChem 13 (2012) 99-107, https://doi.org/10.1002/cphc.201100735.

[101] D. Baddeley, M.B. Cannell, C. Soeller, Visualization of localization microscopy data, Microsc. Microanal. 16 (2010) 64-72, https://doi.org/10.1017/ S143192760999122X.

[102] R.P.J. Nieuwenhuizen, S. Stallinga, Visualization and Resolution in Localization Microscopy, Cell Membrane Nanodomains: From Biochemistry to Nanoscopy, (2014).

[103] M. El Beheiry, M. Dahan, ViSP: representing single-particle localizations in three dimensions, Nat. Methods 10 (2013) 689-690, https://doi.org/10.1038/nmeth. 
2566.

[104] P.R. Nicovich, D.M. Owen, K. Gaus, Turning single-molecule localization microscopy into a quantitative bioanalytical tool, Nat. Protoc. 12 (2017) 453-460, https://doi.org/10.1038/nprot.2016.166.

[105] C. Coltharp, X. Yang, J. Xiao, Quantitative analysis of single-molecule superresolution images, Curr. Opin. Struct. Biol. 28C (2014) 112-121, https://doi.org/ 10.1016/j.sbi.2014.08.008.

[106] S. Malkusch, M. Heilemann, Extracting quantitative information from single-molecule super-resolution imaging data with LAMA - LocAlization Microscopy Analyzer, Sci. Rep. 6 (2016) 34486, https://doi.org/10.1038/srep34486.

[107] F. Levet, E. Hosy, A. Kechkar, C. Butler, A. Beghin, D. Choquet, et al., SR-Tesseler: a method to segment and quantify localization-based super-resolution microscopy data, Nat. Methods 12 (2015) 1065-1071, https://doi.org/10.1038/nmeth.3579.

[108] M. Georgieva, D.I. Cattoni, J.-B. Fiche, T. Mutin, D. Chamousset, M. Nöllmann, Nanometer resolved single-molecule colocalization of nuclear factors by two-color super resolution microscopy imaging, Methods 105 (2016) 44-55, https://doi. org/10.1016/j.ymeth.2016.03.029.

[109] S.V. Pageon, P.R. Nicovich, M. Mollazade, T. Tabarin, K. Gaus, Clus-DoC: a combined cluster detection and colocalization analysis for single-molecule localization microscopy data, Mol. Biol. Cell 27 (2016) 3627-3636, https://doi.org/10.1091/ mbc.E16-07-0478.

[110] T. Lagache, A. Grassart, S. Dallongeville, O. Faklaris, N. Sauvonnet, A. Dufour, et al., Mapping molecular assemblies with fluorescence microscopy and objectbased spatial statistics, Nat. Commun. 9 (2018) 698, https://doi.org/10.1038/ s41467-018-03053-x.

[111] J. Broeken, H. Johnson, D.S. Lidke, S. Liu, R.P.J. Nieuwenhuizen, S. Stallinga et al., Resolution improvement by 3D particle averaging in localization microscopy, Methods Appl. Fluoresc. 3 (2015), https://doi.org/10.1088/2050-6120/3/ $1 / 014003$.
[112] R.F. Laine, A. Albecka, S. van de Linde, E.J. Rees, C.M. Crump, C.F. Kaminski, Structural analysis of herpes simplex virus by optical super-resolution imaging, Nat. Commun. 6 (2015) 5980, https://doi.org/10.1038/ncomms6980.

[113] D. Salas, A. Le Gall, J.-B. Fiche, A. Valeri, Y. Ke, P. Bron, et al., Angular reconstitution-based 3D reconstructions of nanomolecular structures from superresolution light-microscopy images, PNAS 12 (2017) 201704908, https://doi.org/ 10.1073/pnas.1704908114.

[114] H. Heydarian, F. Schueder, M.T. Strauss, B. van Werkhoven, M. Fazel, K.A. Lidke, et al., Template-free 2D particle fusion in localization microscopy, Nat. Methods 15 (2018) 781-784, https://doi.org/10.1038/s41592-018-0136-6.

[115] C. Sieben, N. Banterle, K.M. Douglass, P. Gönczy, S. Manley, Multicolor singleparticle reconstruction of protein complexes, Nat. Methods 15 (2018) 777-780, https://doi.org/10.1038/s41592-018-0140-x.

[116] U. Endesfelder, S. Malkusch, F. Fricke, M. Heilemann, A simple method to estimate the average localization precision of a single-molecule localization microscopy experiment, Histochem. Cell Biol. 141 (2014) 629-638, https://doi.org/10.1007/ s00418-014-1192-3.

[117] N. Banterle, K.H. Bui, E.A. Lemke, M. Beck, Fourier ring correlation as a resolution criterion for super-resolution microscopy, J. Struct. Biol. 183 (2013) 363-367, https://doi.org/10.1016/j.jsb.2013.05.004.

[118] R.P.J. Nieuwenhuizen, K.A. Lidke, M. Bates, D.L. Puig, D. Grünwald, S. Stallinga, et al., Measuring image resolution in optical nanoscopy, Nat. Methods 10 (2013) 557-562, https://doi.org/10.1038/nmeth.2448.

[119] E.F. Fornasiero, F. Opazo, Super-resolution imaging for cell biologists: concepts, applications, current challenges and developments, BioEssays 37 (2015) 436-451, https://doi.org/10.1002/bies.201400170.

[120] A. Burgert, S. Letschert, S. Doose, M. Sauer, Artifacts in single-molecule localization microscopy, Histochem. Cell Biol. 144 (2015) 123-131, https://doi.org/10. 1007/s00418-015-1340-4. 